\title{
Les frontières psychanalytiques du moi : Freud, Klein, Winnicott, Lacan
}

The psychoanalytical boundaries of the Ego: Freud, Klein, Winnicott, Lacan

Thomas Lepoutre 1*, Isabel Fernandez 2, Fanny Chevalier 3, Marie Lenormand 4 , Nicolas Guérin 5

1 Maître de Conférences, EA 3278, Aix Marseille Univ, LPCPP - Maison de la Recherche, 29 Avenue Robert Schuman, 13621 Aix-en-Provence cedex 1, France.

2 Chargée d'enseignement, EA 3278, Aix Marseille Univ, LPCPP - Maison de la Recherche, 29 Avenue Robert Schuman, 13621 Aix-en-Provence cedex 1, France.

3Maître de Conférences, EA 3278, Aix Marseille Univ, LPCPP - Maison de la Recherche, 29 Avenue Robert Schuman, 13621 Aix-en-Provence cedex 1, France.

4 Maître de Conférences, EA 3278, Aix Marseille Univ, LPCPP - Maison de la Recherche, 29 Avenue Robert Schuman, 13621 Aix-en-Provence cedex 1, France.

5 Maître de Conférences HDR, EA 3278, EA 3278, Aix Marseille Univ, LPCPP Maison de la Recherche, 29 Avenue Robert Schuman, 13621 Aix-en-Provence cedex 1, France.

* Auteur correspondant : M. Thomas Lepoutre

Adresse e-mail : thomas.lepoutre@univ-amu.fr

Toute référence à cet article doit porter mention : Lepoutre T, Fernandez I, Chevalier F, Lenormand M, Guérin G. Les frontières psychanalytiques du moi : Freud, Klein, Winnicott, Lacan. Evol psychiatry 2019; 84 (1): pages (pour la version papier) ou URL [date de consultation] (pour la version électronique) 


\section{Résumé}

Objectifs. - Parce que les frontières communément admises du moi et du non-moi se trouvent naturellement fragilisées par l'expérience de la psychanalyse, comme le révèle d'emblée l'adage freudien Wo Es war, soll Ich werden, le présent texte entend présenter la cartographie des problématiques du moi et de ses frontières dans la théorie psychanalytique. L'enjeu en est à la fois de préciser le portrait métapsychologique du moi, tel qu'il se dessine, en sa genèse, ses contours, ses limites, chez les principaux représentants de la théorie psychanalytique, et de montrer en quoi cette même théorie renouvelle par ailleurs un certain nombre de problématiques dans l'abord du moi.

Méthode. - Pour ce faire, en plus d'une interrogation portant sur le corpus fondateur de Freud, l'article confronte, en un même espace, la pluralité des pistes développées par trois référents majeurs dans le champ analytique - Melanie Klein, Donald Winnicott et Jacques Lacan. Si Freud introduit en effet un certain nombre d'interrogations décisives relatives aux limites du moi, une lecture comparative de Klein, de Winnicott et de Lacan, met en valeur ce qui s'en trouve confirmé et prolongé, ce qui s’en trouve infléchi et révisé, ce qui s’en trouve dépassé et renouvelé.

Résultats. - L'examen du corpus freudien permet de problématiser la situation du moi auquel se trouvent assignées ces triples limites 1) intrasubjectives, en tant que le moi est ouvert sur le ça mais séparé « de manière tranchée » du refoulé ; 2) extra-subjectives, en tant que le moi se constitue en s'opposant à la réalité du monde extérieur ; 3) intersubjectives, en tant que le moi se vit, dans son rapport à l'autre, comme séparé de l'objet tout en étant l'objet d'une série d'identifications constituantes. Dans ces trois directions, les limites se trouvent profondément remaniées par les apports de Klein, de Winnicott et de Lacan, qui renouvèlent, chacun à leur manière, la problématique du moi et du non-moi selon qu'ils privilégient et prolongent telle ou telle des intuitions de Freud. 
Discussion. - Indiquer entre ces quatre auteurs les grandes convergences, et creuser par ailleurs leurs différences, permet de marquer l'originalité respective de chacune de leurs contributions. C'est aussi la continuité insistante dans la théorie psychanalytique de certaines problématiques relatives à la séparation entre l'instance du moi et le sujet de l'inconscient, entre le moi et la réalité, entre le dehors et le dedans, entre le sujet et l'objet, entre l'ego et l'alter ego, qui s'en trouve éclairée.

Conclusions. - C'est finalement l'éclatement du Ich freudien et la dispersion des problématiques qui s'ensuit chez les post-freudiens privilégiant chacun telle ou telle piste en vertu des spécificités de leurs paradigmes cliniques, qui permettent de concevoir pleinement l'étendue du problème du moi dans la psychanalyse.

Mots-clés : Moi ; Objet ; Topique ; Théorie ; Psychanalyse ; Freud S. ; Klein M. ; Winnicott DW. ; Lacan J.;

\footnotetext{
Abstract

Objectives. - The commonly accepted boundaries between the ego and the non-ego are naturally weakened by the experience of psychoanalysis, as is revealed by the Freudian Wo Es war, soll Ich werden. This article sets out to map the boundaries of the ego in psychoanalytic theory. The aim is two-fold. First, to provide a metapsychological portrait of the ego by way of a consideration of its genesis, its outlines and its limits as theorized by the main authors of the analytic corpus. Second, to show how this theory can provide new insights in approaching the concept of the ego.

Method. - Besides considering Freud's work, this article weaves together three threads by reappraising the writings of Melanie Klein, Donald Winnicott and Jacques Lacan. While Freud raises a number of crucial points relating to the boundaries of the ego, a comparative reading of these three authors highlights how their work reconsiders, elaborates on or goes beyond Freud's views.
} 
Results. - From a reading of the Freudian corpus emerges a three-fold definition of the boundaries of the ego: 1) Intra-subjective: the ego opens onto the id, but is strictly separate from the repressed 2) Extra-subjective: the ego is established through opposition to external reality. 3) Inter-subjective: the ego, as it relates to the other, is experienced both as separated from the object while at the same time being the object of a series of identifications. Each in their own way, Klein, Winnicott and Lacan shed new light on these questions according to their particular readings of different aspects of Freud's work.

Discussion. - The aim of this paper is to underline the originality of these four authors by pointing out what they have in common and what differentiates them. It also sheds light on a whole set of questions (the separation between the ego as agency and the subject of the unconscious, between the ego and reality, between the outside and the inside, between the subject and the object, between the ego and the alter ego) that pervade psychoanalytical theory.

Conclusions. - Ultimately it is the shattered state of the Freudian Ich - as revealed by its diverse readings by the post-Freudians according to their respective clinical paradigms - that is the key to understanding the extent of the problem of the ego in psychoanalysis.

Keywords: Ego; Object; Topography; Metapsychology; Psychoanalysis; Freud S.; Klein M.; Winnicott DW.; Lacan J.; Self; Ich; Reality; Subject of the unconscious 


\section{Introduction}

On connaît la formule par laquelle s'annonce, de façon un peu solennelle, le tranchant de la découverte freudienne :

«Rien d'étranger n'est entré en toi ; c'est une partie de ta propre vie d'âme qui s'est soustraite à ta connaissance et à la domination de ta volonté. C'est d'ailleurs pourquoi tu es si faible dans la défense ; tu combats avec une partie de ta force contre l'autre partie, tu ne peux pas rassembler toute ta force comme contre un ennemi extérieur. Et ce n'est même pas la pire ni la moins importante part de tes forces animiques qui est entrée ainsi en opposition avec toi et est devenue indépendante de toi. » ([1], p. 49-50)

Tel est en effet le sens de la « révolution » opérée par Freud, consistant, après Copernic et sa critique du géocentrisme, après Darwin et sa critique de l'anthropocentrisme, à jeter un soupçon définitif sur l'ego-centrisme, en soulignant que «le moi n'est pas maître dans sa propre demeure ».

Par la remise en cause de cette hégémonie et la promotion du sujet divisé, les frontières communément admises du moi et du non-moi se trouvent naturellement fragilisées par l'expérience de la psychanalyse - et ce dès les premières contributions de Freud. Dès les Études sur l'hystérie, la mise au jour de l'inconscient passait par la découverte de ce «corps étranger» interne à la subjectivité, auquel devait être ramené tout ce qui fait échec à la «toute-conscience » du moi - lequel est régulièrement hanté, à son insu, par quelque chose dont il ne veut rien savoir mais qui néanmoins lui appartient (c'est d'ailleurs là la «formule psychologique » de la possession démoniaque) : «La psyché clivée est ce démon dont la naïve observation des temps anciens, superstitieux, croyait les malades possédés. Le fait qu'un esprit étranger à la conscience 
vigile du malade règne en lui est exact, sauf que ce n'est pas un esprit vraiment étranger, mais une part de son propre esprit. » ([2], p. 277)

Où passe la limite entre ce qui est propre au moi et ce qui lui est étranger, dès lors qu'une « inquiétante étrangeté » travaille le fonctionnement du moi lui-même, en le hantant comme le démon ? La réponse n'est pas si tranchée pour Freud - à preuve la limite de cette métaphore du « corps étranger » elle-même, laquelle n'est bientôt plus à même de donner à penser l'intrication du refoulé et du moi, ou, pour mieux dire, l'infiltration du moi par le refoulé :

«notre groupe psychique pathogène ne se laisse pas dégager du moi avec précision, ses strates externes se fondent de tous côtés dans des éléments du moi normal [...]. La limite entre les deux devient dans l'analyse purement conventionnelle, mise tantôt ici, tantôt là, ne se laissant à certains endroits pas même repérer. L'organisation pathogène ne se comporte pas vraiment comme un corps étranger, mais bien plutôt comme un infiltrat. » ([2], p. 277)

Que la délimitation paraisse à Freud «conventionnelle» tant elle s'efface suffit à légitimer une interrogation sur les frontières du moi, telles qu'elles se dessinent dans la psychanalyse. Interrogation double, en réalité, puisqu'en regard de la polysémie du mot, les frontières en cause peuvent regarder aussi bien les limites du moi comme instance spécifique à l'intérieur de l'appareil psychique, que les limites, plus générales sinon plus assurées, du moi vis-à-vis de l'objet, pour reprendre dans les coordonnées de la psychanalyse la classique opposition entre le sujet et le monde extérieur. Les deux problématiques doivent sans doute être traitées dans la solidarité où les tient cette polysémie, puisque Freud lui-même les tient souvent indistinctes de façon féconde, comme nous allons le voir. En outre, la situation privilégiée du moi dans son rapport à l'autre comme objet d'identification mérite sans doute un développement 
supplémentaire, dans la mesure où la nature du moi comme précipité identificatoire s'en trouve spécifiée.

Pour ce faire, en plus d'une interrogation sur le corpus fondateur, on entendra restituer et confronter, en un même espace, la pluralité des pistes développées à ce sujet par trois référents majeurs dans le champ analytique, qui balisent assez légitimement l'espace de cette problématique. Si Freud introduit en effet un certain nombre d'hypothèses décisives, il convient de les mettre également en perspective en recourant, dans l'œuvre de Melanie Klein, de Donald W. Winnicott et de Jacques Lacan, à ce qui s'en trouve confirmé et prolongé, ce qui s'en trouve infléchi et révisé, ce qui s’en trouve dépassé et renouvelé.

L'enjeu d'un tel exercice consiste à y fixer schématiquement les grandes convergences, mais aussi à y creuser les différences, pour marquer l'originalité respective de chacune des contributions. Cela revient à préciser les contours du moi tel qu'il se dessine dans la conception de Freud, mais également à penser les pistes qu'elle suggère tout en laissant à d'autres le soin de les développer. Des hypothèses inédites relatives au développement du moi précoce autour d'un «bon objet interne » chez Klein, à la promotion du self et du rôle de l'environnement dans sa constitution chez Winnicott, en passant par le recentrement des problématiques du moi sur sa dimension d'image spéculaire chez Lacan, c'est en effet les virtualités conceptuelles du Ich freudien qui se trouvent prolongées, ou dépassées, et dont il faut rendre compte. C'est que le «moi » de Freud fonctionne décidément comme un foyer d'agrégation d'interprétations divergentes pour les psychanalystes, ce dont attestent notamment les multiples traductions proposées par les post-freudiens - moi, self, ego, etc. -, où le choix sémantique est chaque fois l'indice d'un remaniement théorique implicite du concept engageant l'ensemble de l'édifice psychanalytique. En quoi c'est finalement l'éclatement du Ich freudien et la dispersion des 
problématiques qui s'ensuit chez les post-freudiens, qu'il convient de rassembler en un même espace concevoir pleinement l'étendue du problème du moi dans la psychanalyse.

\section{Avec Freud. Le moi comme « être de frontière »}

On sait que Freud fait coïncider le point d'origine de la psychanalyse avec la découverte du sens du symptôme comme « ce qu'il y a de plus étranger au moi » : «Le symptôme est issu du refoulé, il en est pour ainsi dire le représentant devant le moi, mais le refoulé est pour le moi territoire extérieur, territoire extérieur interne, tout comme la réalité est - permettez-moi cette expression inhabituelle - territoire extérieur externe. » ([3], p. 140)

Prendre acte de ces frontières doubles, ouvertes sur le territoire intérieur d'une part, sur le territoire extérieur d'autre part, nous invite donc à désigner les lieux d'émergence du «moi » dans l'écrit freudien où on le voit 1) se dessiner autant comme instance, délimitée à l'intérieur de l'appareil psychique, que 2) problématisé comme vécu, tel qu'il est subjectivement ressenti, sur le mode de l'évidence naturelle, en tant que séparé du monde de la prétendue «réalité extérieure ». Ceci rappelé, cela doit nous permettre 3) de le situer précisément, dans un troisième temps, dans son rapport d'aliénation à l'objet.

\subsection{Le moi et le refoulé : limites topiques et frontières diplomatiques}

Lorsqu'on envisage la situation du moi dans les premiers écrits de Freud - disons, ceux allant des Études sur l'hystérie jusqu'à la Traumdeutung -, il est bien difficile d'en isoler une problématique précise, aux contours arrêtés - a fortiori parce que le «moi » ne prend 
naturellement son acception technique d'instance qu'avec l'avènement de la seconde topique, à la suite du tournant de 1920. S'y trouvent néanmoins avancées de façon précoce certaines intuitions qui se retrouveront ultérieurement comme des motifs récurrents du discours freudien.

La première d'entre elles, déjà esquissée en introduction et fermement établie dès les Études de 1895, est assurément qu'au plan dynamique le moi, en tant qu'il prend en charge le pôle défensif du conflit psychique, est d'abord ce qui s'oppose au refoulé - soit ce noyau de représentations considérées comme «inconciliables» avec «la masse de représentations dominante du moi », et vis-à-vis desquelles ce dernier cède « tout à l'envie de se défendre » ([2], p. 304). La conséquence clinique en est qu'il constitue d'abord une instance de méconnaissance au service du refoulement, ne pouvant être qu'une conscience étroite pour le sujet vis-à-vis d'une vérité inconciliable et inconsciente à lui-même, dont il est séparé par «clivage». La conséquence théorique, formalisée au plan économique dans l' «Esquisse », est qu'il ne peut être que «perturbateur» sur l'éconduction de l'énergie psychique, ne sachant, dans sa fonction de régulation des quantités d'excitation responsables de plaisir et de déplaisir, «qu'inhiber les processus psychiques primaires » ([4], p. 632). D’un point de vue topique, il se dégage alors déjà comme une « organisation » autonome et s'isole comme un réseau à investissement constant (un « groupe de neurones qui est investi de façon constante ») ([4], p. 631) -encore que les frontières en soient mouvantes. D'un point de vue génétique, enfin, il est progressivement investi du rôle de garant du «signe de réalité » permettant de faire la différence, dans l'horizon de l'expérience de satisfaction, entre sa perception et sa répétition hallucinatoire ([4], p. 634).

Ces indications précoces attestant d'un premier clivage entre l'inconciliable du refoulé et le moi, tous deux caractérisés des principes de fonctionnement économique hétérogènes l'un à l'autre, sont prolongées dans l'Interprétation du rêve. C'est alors « la scission entre l'inconscient 
et le conscient - le refoulé et le moi - qui est mise à nu» ([5], p. 611) et qui explique le processus formateur du rêve 1 . Si cette «scission» explique qu'il s'éveille «en moi » des pensées inconscientes que pourtant je ne reconnais pas et qui m'habitent à mon insu, en sorte que sous l'effet de la censure «ça pense » plus en moi que je ne le sais, c'est que le sujet du rêve méconnait une partie de son propre désir, qui s'articule justement à l'endroit où son moi «n'y est pas » : manière de faire déjà pressentir, selon la formule de Lacan, que le Moi n'est toujours, au mieux, que « la moitié du sujet » ([6], p. 346).

Si entre 1900 et 1911 Freud confirme çà et là que certaines représentations sont refoulées parce qu'elles s'opposent à d'autres, plus fortes, «pour lesquelles nous employons le concept global de "moi", composé chaque fois différemment »([7], p. 181), c'est en réalité à partir de la période 1914-1915 que le moi acquiert progressivement son véritable statut métapsychologique. L'introduction du narcissisme permet en effet de dégager tout à la fois :

1) que le moi n'est pas constitué d'emblée, qu'«il n'existe pas dès le début, dans l'individu, une unité comparable au moi » ([8], p. 221). Il faut donc en inférer une généalogie du $m o i$, lequel n'apparaît pas tant comme le résultat d'une différenciation progressive, mais exige le concours d'une «nouvelle action psychique » qui le précipite à partir de l'autoérotisme ;

2) que sa situation comme lieu de la défense dans le conflit psychique est simultanément amendée et confirmée. Amendée, parce qu'étant constitué comme une stase de libido, s'offrant lui-même comme objet d'amour à la sexualité, le moi ne s'oppose plus au sexuel par nature, il en est aussi un objet ; au vieux conflit entre le moi et la sexualité se substitue une perspective où le

1 Tout du moins à partir de l'édition de 1919, qui réintroduit cette «scission» au cœur d'une œuvre qui ne différenciait à l'origine que les systèmes Inconscient, Préconscient, Conscient, dans le cadre d'un « appareil » où la notion de «moi » n'intervenait de façon explicite que de façon sporadique. 
moi est lui-même gonflé par le sexuel. Et confirmée cependant, parce que, même s'il n'existe plus de lieu par nature innocent dans la psyché, son rôle dans le refoulement s'en trouve précisé : «Le refoulement, avons-nous dit, provient du moi ; nous pourrions préciser : de l'estime de soi qu'a le moi. [...] La formation d'idéal serait du côté du moi la condition du refoulement » ([8], p. 236).

3) enfin, que le moi comme instance est lui-même susceptible d'être composé de formations satellites, qui peuvent s'en détacher comme instances plus ou moins différenciées, et qui en règlent le comportement - ainsi de l'idéal du moi et de cette «instance psychique particulière qui remplit la tâche de veiller à ce que soit assurée la satisfaction narcissique provenant de l'idéal du moi, et qui, dans cette intention, observe sans cesse le moi actuel et le mesure à l'idéal » ([8], p. 236). L'introduction du narcissisme permet ainsi de «pénétrer plus avant dans la structure du moi » pour reconnaître par exemple dans cet idéal le fameux «censeur du rêve », et préciser le fonctionnement des «tendances refoulantes dominant le moi ». La clinique de la mélancolie soutient en outre cette idée qu'une instance particulière est susceptible de se spécifier dans le moi, en sorte qu'une partie du moi se pose face à une autre pour la juger et l'attaquer - ouvrant la voie à la reconnaissance du surmoi et la formalisation de la seconde topique.

C'est en effet, comme on sait, en 1923 que Freud parvient finalement à «achever l'édification de notre représentation du moi », et que ses «frontières » topiques trouvent leur expression métapsychologique définitive. Le schéma en est connu, il décrit qu' « un individu est donc pour nous un ça psychique, non connu et inconscient, sur lui se trouve posé en surface le moi, développé à partir du système- $P c$ comme noyau. Si nous cherchons à présenter graphiquement les choses, alors nous ajouterons que le moi n'enveloppe pas le ça totalement, 
mais seulement dans la mesure où le système $P c$ en forme la surface, donc à peu près comme le disque germinal est posé sur l'œuf. Le moi n'est pas séparé de manière tranchée du ça, il conflue avec lui vers le bas. » ([9], p. 268)

Les difficultés inhérentes à une telle figuration tiennent à ce que les limites internes du moi s'y trouvent à la fois atténuées et confortées : atténuées du côté du ça, puisqu'il n'en est qu'une «partie modifiée », soit cette organisation différenciée et spécifiée sous l'effet du monde extérieur ; et confortées, sinon radicalisées, du côté du refoulé, puisqu'il en est «démarqué de manière tranchée » par la barrière des résistances et le clivage du refoulement.

Cette double frontière - faite d'ouverture, sur une frange, vis-à-vis du ça, et de fermeture, sur un bord, vis-à-vis du refoulé - reconduit en fait la coupure radicale inaugurée par le refoulement pour mieux rendre compte des résistances insurmontables que le moi manifeste à «s'occuper du refoulé », mais entend aussi prendre acte de cette nouveauté, que le moi est pour une grande part inconscient ([9], p. 262). «Une partie du moi aussi - et combien cette partie du moi est importante, seul un dieu le sait - peut être ics, est sûrement ics » : telle est l'extension inédite du moi dans la seconde topique, qui en rend la frontière en son genre abyssale - puisque le moi soumet «à son influence des circonscriptions toujours plus grandes et des strates plus profondes du ça ».

On comprend alors le sens de l'avertissement freudien, lorsque l'on envisage une telle « décomposition de la personnalité », de ne pas croire «à des frontières tranchées, telles qu'elles ont été tracées artificiellement en géographie politique. Nous ne pouvons faire droit à la spécificité du psychique par des contours linéaires comme dans le dessin ou dans la peinture primitive, mais plutôt par des champs de couleur qui se fondent comme chez les peintres modernes » ([3], p. 162). C'est la métaphore définitive exprimant l'effacement de la limite entre 
l'instance du moi et le royaume du ça, sinon l'inanité d'une démarcation artificielle entre le moi qui s'identifie et celui qui « est pensé » par l'inconscient.

L'intérêt théorique de cet «élargissement » du moi consiste à mieux rendre compte, au plan clinique, des effets de duperie 2 du moi immanquablement générés par sa triple servitude au ça, au surmoi et à la réalité, où il a essentiellement fonction de méconnaissance, de rationalisation, de défense compulsive contre les commandements inconscients, et d'où il entend sortir avec le «beau rôle » de l'homme d'État 3 qui fait croire qu'il commande, ou du cavalier 4 suivant son cheval pour donner l'illusion qu'il a la main.

Contre cette fausse prétention et ce semblant d'autonomie, la réalité clinique de la névrose montre plutôt l'assujettissement auquel le moi est soumis dans sa dépendance aux instances inconscientes qui l'asservissent, lui qui ne peut jouer en retour que « le rôle ridicule qui est au cirque celui du stupide Auguste, prétendant par ses gestes donner aux spectateurs la conviction que tous les changements qui ont lieu sur la piste s'effectuent à son seul commandement. Mais seuls les plus jeunes des spectateurs lui accordent foi. » ([10], p. 300). C'est que le «pauvre moi »n'apparaît plus seulement contesté, sur ses frontières, par trois despotes sévères qui lui disputent sa maîtrise et son étendue, mais s’institue également lui-même comme un «être de frontière »

2 On se souvient du portrait qu'en fait Freud : «flagorneur, opportuniste et menteur », ce « valet soumis qui cherche l'amour de son maître » entreprend avant tout de « rester en bonne entente avec le ça, revêt les commandements ics de ses rationalisations pcs, fait miroiter l'illusion que le ça obéit aux avertissements de la réalité même là où le ça est resté rigide et inflexible, maquille les conflits du ça avec la réalité » ([9], p. 299)

3 «Dans sa position intermédiaire entre ça et réalité, il ne succombe que trop souvent à la tentation de devenir flagorneur, opportuniste et menteur, un peu comme un homme d'État qui, bien qu'ayant une bonne intelligence de la situation, veut néanmoins s'affirmer dans la faveur de l'opinion publique. » ([9], p. 299)

4 «Ainsi il ressemble, dans son rapport avec le ça, au cavalier qui doit brider la force supérieure du cheval, avec cette différence que le cavalier tente cela avec ses propres forces, le moi avec des forces empruntées. Cette comparaison nous emporte un peu plus loin. De même qu'il ne reste souvent rien d'autre à faire au cavalier, s'il ne veut pas se séparer de son cheval, que de le conduire là où il veut aller, ainsi le moi a coutume de transposer en action la volonté du ça, comme si c'était la sienne propre. » ([9], p. 270). 
servant essentiellement comme un médiateur d'exigences contradictoires : "Comme être de frontière, le moi veut faire l'intermédiaire entre le monde et le ça, rendre le ça docile au monde et rendre le monde, par le moyen de ses actions musculaires, conforme au souhait-du-ça. » ([9], p. 299)

On comprend qu'au terme de son enseignement, Freud en revienne à cette conclusion définitive de la psychanalyse formulée dans Malaise, qui dénonce dans l'autonomie et les frontières du moi un leurre de la subjectivité, laquelle n'est jamais réductible à l'évidence de son identité apparente :

« Normalement, rien n'est pour nous plus assuré que le sentiment de notre soi, de notre moi propre. Ce moi nous apparaît autonome, unitaire, bien démarqué de tout le reste. Que cette apparence soit un leurre, qu'au contraire le moi se continue vers l'intérieur, sans frontière tranchée, dans un être animique inconscient que nous qualifions de ça, auquel il sert en quelque sorte de façade, c'est ce que nous a enseigné, la première, la recherche psychanalytique. [...] Mais, vers l'extérieur au moins, le moi semble affirmer des frontières claires et tranchées. » 5 ([11], p. 251)

Avoir redessiné ainsi l'ambiguïté des limites internes de cet «être de frontière » ouvre alors un nouveau front, sur lequel le moi est destiné à «représenter les exigences du monde extérieur ». Entendons que si, dans cette première perspective, le moi apparaît à Freud comme le produit d'une différenciation progressive du ça résultant de sa relation à la réalité, il reste à penser sa genèse au plan de la différenciation moi - monde extérieur.

\footnotetext{
5 En insert, on remarquera qu'en jetant ainsi un soupçon radical sur l'évidence bien assurée du « sentiment du moi » qui fait systématiquement méconnaître l'inconscient, Freud l'interroge certes comme leurre, mais le prend par ailleurs comme une certitude vécue qui n' aurait pas à être questionnée et expliquée dans sa genèse : or on sait que ce sentiment de continuité d'existence n'est pas si assuré qu'il interdise toute réflexion psychopathologique à son sujet, comme le démontrera toute l'œuvre de Winnicott. Voir infra.
} 


\subsection{Le moi et la réalité : espace interne, frontières externes}

On sait que cette seconde perspective se thématise, dans l'œuvre de Freud, avec la prise en compte du développement du principe de réalité, lequel apparaît lorsque «l'appareil psychique dut se résoudre à représenter l'état des faits réel du monde extérieur et à tendre à la modification réelle », là où originairement, « le pensé (le souhaité) fut tout simplement posé de façon hallucinatoire » ([12], p. 14). L'opposition entre principe de plaisir et principe de réalité, et la nécessaire soumission du premier au second, se traduisent en effet pour Freud dans un certain mode de fonctionnement du moi, passant d'un «moi-plaisir », qui hallucine la satisfaction de ses besoins et méconnaît la réalité du monde extérieur avec laquelle il tend à se confondre dès lors qu'elle est plaisante, à un «moi-réel », pour lequel n'était «plus représenté ce qui était agréable, mais ce qui était réel, même si cela devait être désagréable ».

Cette progressive «mutation du moi-plaisir en moi-réel » ([12], p. 19) invite à penser que la réalité du monde extérieur, pour le moi, n'est jamais donnée à l'origine, mais construite et appréhendée à partir d'une «déception» fondamentale. Freud l'évoque, tout en laissant à d'autres, comme Winnicott, le soin de donner à cette perspective toute son importance (voir infra), mais la séparation du «moi-plaisir» et du monde extérieur présuppose sans doute d'autres séparations, le nourrisson qui « hallucine vraisemblablement l'accomplissement de ses besoins internes » ne pouvant mettre fin à la domination du principe de plaisir «qu'avec le plein détachement psychique d'avec les parents » ([12], p. 15) - façon de dire que la «réalité » n'est jamais que la réalité de la perte. Mais cela suppose en outre que le moi y fasse droit «en dehors des qualités de plaisir et de déplaisir », toute déplaisante qu'elle soit, en reconnaissant la conformité du représenté avec la réalité - c'est là la fonction du «jugement de réalité » qu'il 
prend en charge. Du reste, s'il faut au moi un «principe de réalité », c'est bien parce que la réalité ne fait pas principe : celle-ci ne s'impose que le long d'un développement du moi qui ne peut se la représenter, pour Freud, qu'à la condition que la satisfaction immédiate promise par le principe de plaisir se trouve reportée à son horizon 6 .

Le réexamen métapsychologique, en 1915, de cette généalogie du moi d'abord gonflé de plaisir puis faisant la part du monde extérieur, permet de la préciser et de la compléter dans « Pulsions et destins des pulsions ». Freud y confirme l'idée que la genèse de l'opposition sujet (moi) - objet (monde extérieur) est corrélative de l'opposition plaisir-déplaisir selon la séquence esquissée, mais il en complexifie la linéarité, en y introduisant une étape supplémentaire. Freud y souligne en effet qu'en une étape originaire, le nourrisson dans l'Hilflosigkeit est pourtant d'emblée en mesure d'effectuer la «différenciation » fondatrice : «l'opposition de moi - nonmoi (extérieur), (sujet - objet), est imposée précocement à l'être individuel, par l'expérience qu'il a de pouvoir faire taire les stimuli externes par son action musculaire, tout en étant sans défense contre les stimuli pulsionnels. » ([13], p. 181)

Aptitude à fuir l'excitation externe, contre éprouvé de continuité de l'excitation interne : telle est la tension originaire où l'être trouve pour Freud un premier « point d'appui pour séparer un “à l'extérieur" d'un “à l'intérieur” » ([13], p. 167). Il faut dégager la portée, au plan métapsychologique, de ce « critère objectif » et originaire, selon le mot de Freud, de démarcation entre le moi et le non-moi : c'est qu'il y a des excitations contre lesquelles mon corps peut quelque chose, que l'infans « met au compte d'un monde extérieur »; des excitations contre

6 On vérifie ici, en insert, que la «substitution » par le principe de réalité ne revient pas purement et simplement à une «destitution du principe de plaisir» : elle s'accomplit bien plutôt comme une modification de ce dernier, pour autant qu'après son instauration, le principe de réalité vient encore, in fine, à la satisfaction du principe de plaisir. C'est que le principe de réalité, par lequel le moi cesse de se confondre dans le cercle étroit et immédiat du «moiplaisir » pour faire droit au monde extérieur, ne s'établit qu'à la condition de reprogrammer à son horizon la satisfaction - ce qui ouvre déjà la voie à la reconnaissance que la « réalité » n'est jamais que ce qui est perçu à travers la «fenêtre » du fantasme... 
lesquelles mon corps ne peut rien, qui deviennent «le signe caractéristique d'un monde intérieur »- Freud faisant ainsi entendre que c'est d'abord la pulsion qui découpe un intérieur et un extérieur du corps, et l'éprouvé de sa «constance » qui fait la séparation, pour le moi, entre un dedans et un dehors 7 .

En lien direct avec ce qui précède, on s'avise alors d'une inversion que Freud fait subir désormais aux deux termes, «moi-plaisir » et «moi-réel », vis-à-vis de la succession décrite quatre ans auparavant dans les «Formulations sur les deux principes ». C'est un hapax méconnu dans l'œuvre freudienne, puisqu'il n'y reviendra pas, mais dans la genèse complétée que Freud propose en 1915, il considère en effet que le moi «se change à partir du moi-réel initial, qui a différencié intérieur et extérieur selon un bon critère objectif, en un moi-plaisir purifié qui place le caractère de plaisir au-dessus de tout autre » ([13], p. 183). L'inversion, en forme de chiasme, vient de ce qu'entre les deux textes, le «moi-réel » a changé d'acception pour Freud : il n'est plus à la fin, coïncidant avec l'instauration du principe de réalité, mais au début, avant même que « sous la domination du principe de plaisir, s'effectue en lui un nouveau développement » vers le moi-plaisir ; le moi-plaisir, lui, à l'inverse, n'est lui plus au début mais en position seconde, à partir du moment où «il accueille dans son moi les objets offerts, dans la mesure où ils sont sources de plaisir, il s'introjecte ceux-ci et, d'un autre côté, expulse hors de lui ce qui, dans son intérieur propre, lui devient occasion de déplaisir. » ([13], p. 182)

Tout se passe alors comme si Freud considérait finalement que la domination du principe de plaisir, non seulement n'était pas la première étape, mais pouvait de plus marquer un recul et

$7 \mathrm{Au}$ passage, cela éclaire sans doute une formule ultérieure, exprimée par Freud sous une forme énigmatique, posant que le moi se constitue comme une image projetée du corps : «Le moi est avant tout un moi corporel, il n'est pas seulement un être de surface, mais lui-même la projection d'une surface ». Ainsi que la note autorisée de la Standard Edition qui la complète : «Le moi est finalement dérivé de sensations corporelles, principalement de celles qui ont leur source dans la surface du corps. Il peut ainsi être considéré comme une projection mentale de la surface du corps ». On sait que Lacan insistera sur les rapports de surface existant justement entre la consistance du moi et celle de d'une image spéculaire. 
voiler une acquisition première du moi - le «moi-réel initial » disposant lui, « d'emblée », d'un critère objectif relatif à la différenciation réelle du moi et du monde extérieur. Cela donne à penser, a contrario, le réagencement imaginaire de cette différenciation pour le moi, lorsque s'y mêle le plaisir et qu'elle est ressaisie par le fantasme, dans l'étape seconde, intégralement dominée par le principe de plaisir. Plus aucune appréhension réelle du moi n'est en effet possible, dès lors que le moi et le monde sont scindés, découpés, séparés, selon la ligne du plaisir-déplaisir, via une différenciation intégralement organisée par le jeu des introjections et des projections qui démarquent un monde intérieur à partir de tout ce qui est source de plaisir, et un monde extérieur à partir de tout ce qui est déplaisant - y compris ce qui, du moi, est ressenti comme étranger. On comprend pourquoi «l'externe, l'objet, le haï seraient, au tout début, identiques » ([13], p. 183) - thèse qui culmine dans l'idée que l'objet naît dans la haine, au plan individuel, et qui s'épanouira, au plan collectif, jusque dans le «narcissisme des petites différences ». C'est d'ailleurs dans des termes pratiquement identiques que Freud reprendra, dans son petit texte sur « la négation », cette opposition originaire - en l'alignant, dans le langage des motions pulsionnelles, sur le plan de l'incorporation et de la projection 8 -, ainsi que dans Malaise, qui permettra de préciser que «les frontières de ce moi-plaisir primitif ne peuvent échapper à la rectification par l'expérience. Mainte chose qu'on ne voudrait pas abandonner comme étant dispensatrice de plaisir n'est pourtant pas moi, est objet, et maint tourment qu'on veut jeter au dehors se révèle pourtant comme étant inséparable du moi. » ([11], p. 253)

Si l'instauration du principe de réalité permet par la suite de distinguer ce qui est perçu de ce qui est représenté, en instituant du même coup la différenciation du monde intérieur,

8 «Cela je veux le manger ou bien je veux le cracher, et en poussant plus avant le transfert : cela je veux l'introduire en moi, et cela l'exclure de moi. Donc : ça doit être en moi ou bien hors de moi. Le moi-plaisir originel veut [...] s'introjecter tout le bon, jeter loin de lui tout le mauvais. L'étranger au moi, ce qui se trouve à l'extérieur est pour lui tout d'abord identique. » ([14], p. 168-169). 
subjectivement représenté, et du monde extérieur, objectivement retrouvé, on sait que le moi conservera longtemps les stigmates de cette genèse complexe. Deux figures en un sens opposées mais complémentaires en livrent sans doute les meilleures justifications cliniques. C'est, d'une part, le «sentiment océanique » décrit par Romain Rolland, dans l'horizon duquel l'évidence du moi, vécu de façon étroite, n'apparaît que comme le « reste ratatiné » d'un état où, «à l'origine, le moi contient tout $»-$ à mettre en regard de son développement ultérieur, où le moi se sépare du monde extérieur pour s'enfermer dans l'étroitesse de sa maturité dont «les frontières sont plus resserrées et plus tranchées. ([11], p. 253)

C'est d'autre part le sentiment «d'inquiétante étrangeté » qui nous saisit immanquablement à l'évocation de la figure du double maléfique de nous-même, que Freud nous a appris à interpréter comme le visage où se reflète naturellement ce qui est désavoué et projeté en dehors du moi propre - et qui n'apparaît que par une « régression à des époques où le moi ne s'était pas encore rigoureusement délimité par rapport au monde extérieur et à l'autre... » ([15], p. 170)

\subsection{Le moi et l'objet : identifications et frontières intersubjectives}

Cela fait naturellement transition vers une troisième frontière qui doit s'établir, sur l'axe intersubjectif, entre ce qui relève du moi et ce qui relève de l'autre.

S'agissant de cette limite, la clinique psychanalytique a tôt fait de reconnaître les effets d'assimilation sinon même de confusion existant fréquemment, dans l'identification, entre le moi et son semblable. D'abord dans le symptôme hystérique, qui manifeste souvent une identification symbolique par laquelle les sujets hystériques parviennent à exprimer «les expériences vécues 
d'une grande série de personnes et pas seulement celles qui leur sont propres, à souffrir en quelque sorte pour toute une multitude humaine et à jouer, par leurs seuls moyens personnels, tous les rôles d'un spectacle» ([5], p. 184). Au-delà de ses prétendues propriétés d'imitation, l'hystérie met ainsi en valeur la logique d'appropriation inconsciente du moi vis-à-vis de l'autre, dès lors qu'un élément commun les relie dans l'inconscient, et à travers laquelle le moi espère transférer sur lui-même quelque chose qui est attaché à l'autre.

L'importance de cette identification, où le sujet méconnaît volontiers le «trait commun » qui l'assimile à l'autre, le «de même que » en vertu duquel il se met à la place de l'autre, est d'ailleurs rapidement confirmée, en dehors du symptôme, dans la compréhension que l'on peut avoir, à leur interprétation, des rôles déguisés que jouent les personnages apparaissant dans les rêves. Dans la Traumdeutung, en effet, Freud nous avertit que le «moi » trouve volontiers dans le rêve un nouveau visage, ou plutôt un nouveau masque (ce qui confirme son statut de duperie), la tromperie se révélant en ceci que les rêves «sont dans leur totalité absolument égoïstes ; dans tous, le cher moi entre en scène, bien que déguisé. Les souhaits qui sont accomplis en eux sont régulièrement des souhaits de ce moi ; ce n'est jamais qu'une apparence trompeuse si l'intérêt pour un autre est censé avoir provoqué un rêve » ([5], p. 308). Si le « moi » ne porte pas aussi loin qu'on le croit, il peut aussi se trouver là où on ne l'attend pas. C'est l'une des illusions du moi dénoncée par la psychanalyse, et qui s'exprime mieux la nuit que dans la vie diurne : son aptitude à se « déguiser » en prenant les traits d'un autre, au point que « là où dans le contenu de rêve ce n'est pas mon moi mais seulement une personne étrangère qui apparait, je peux tranquillement supposer que mon moi se cache, par identification, derrière cette personne. » ([5], p. 367). Il faut en tirer toutes les conséquences : si dans les rêves, dans tous les cas, «mea res agitur», «c'est de moi qu'il s'agit» ([5], p. 489), et si tous les «personnages » sont 
susceptibles de représenter en fait le rêveur, c'est qu'il n'y a pas de différenciation opérante dans le rêve entre moi et l'autre, qui se renvoient l'un à l'autre comme en un miroir - au point d'ailleurs qu'à l'inverse «d'autres fois où mon moi apparaît dans le rêve, la situation dans laquelle il se trouve m'enseigne que derrière le moi se cache par identification une autre personne » ([5], p. 367). On peut dire qu'en ces cas, «je est un autre » certes, mais aussi que «l'autre c'est moi », selon un jeu d'identifications qui trouve ici sa première inscription dans l'œuvre de Freud, et qu'il reconnaîtra ultérieurement comme au fondement même du moi.

La consistance identificatoire du moi trouve dans cette propriété à «se cacher » derrière l'autre dans le rêve l'une de ses expressions cliniques durable - mais ce qui est vrai de la vie de rêve l'est sans doute aussi en partie de la vie vigile, et Freud nous apprendra par la suite à reconnaître que le moi propre se cache derrière l'objet, selon des configurations et des mécanismes différents, dans de nombreux contextes psychopathologiques encore. Ainsi de la névrose en général, où le moi reproche si spontanément à l'autre ce qu'il se reproche à lui-même, cherchant à «se défendre contre un auto-reproche en élevant le même reproche contre une autre personne » ([16], p. 215); de même de la mélancolie, où «l'ombre de l'objet est tombée sur le moi » et où par conséquent, à l'inverse, les auto-reproches sont en réalité des reproches adressés à un autre - le surmoi frappant le moi parce qu'il s'est substitué à l'objet perdu par « identification narcissique » [17]; de la psychose 9 encore, où « le jaloux comme le persécuté, [...] projettent vers l'extérieur, sur d'autres, ce qu'ils ne veulent pas percevoir dans leur propre intérieur » ([19], p. 91), et où, au final, «c'est l'ego qui parle par l'alter ego » pour reprendre le mot de Lacan, etc. Quelles que soient les modalités différentielles de ces situations hétérogènes,

9 Sur ce point, relevons qu'il est naturellement tout sauf anodin que l'on doive justement à un auteur longuement engagé dans la psychothérapie des psychoses, P. Federn en l'occurrence, l'une des premières réflexions systématiques sur les «frontières du moi » en psychanalyse - pour reprendre son expression décisive [18]. 
invitant à délimiter précisément l'identification de processus voisins comme l'incorporation, l'introjection, l'intériorisation ou la projection, on entrevoit s'inaugurer là le point de départ d'une problématique importante pour la psychopathologie psychanalytique, s'agissant de faire la part des choses entre le moi et l'autre.

C'est que le rôle de l'identification, cette «assimilation d'un moi à un moi étranger, par suite de laquelle ce premier moi se conduit, à certains égards, comme l'autre, l'imitant, en quelque sorte l'accueillant en soi » ([3], p. 146) est progressivement reconnu par Freud comme central dans la constitution de la vie psychique elle-même. D'abord, avec l'introduction du narcissisme, qui permet à Freud d'avancer que le sujet, ou telle de ses instances, à commencer par l'idéal du moi, est constitué sur le modèle de ses semblables ou ses objets antérieurs ; ensuite dans le Moi et le Ça, où les effets structurants et durables des premières identifications, perceptibles dans le fonctionnement des instances qu'elles spécifient, sont reconnus comme des reliquats, des restes, de relations d'objet abandonnées, des substituts venant à la place d'anciens investissements - le moi lui-même étant in fine constitué en son noyau par une multitude d'identifications idéales à ses imagos originaires. Ainsi Freud statue-t-il à la fin «que le moi, pour une bonne part, se forme à partir d'identifications qui prennent le relais d'investissements du ça laissés vacants, que les premières de ces identifications se conduisent régulièrement comme instance particulière dans le moi, se posent face au moi en tant que sur-moi, alors qu'ultérieurement le moi renforcé peut bien se comporter de façon plus résistante envers de telles influences identificatoires. » ([9], p. 291)

Qu'est-ce à dire, sinon que la constitution du moi lui-même, pour Freud, non seulement s'effectue sur le modèle de l'autre, mais suppose en outre que cet autre qui a été perdu se trouve de nouveau érigé dans le moi ? 
Mais parvenu à ce point où se dessine, à l'horizon de l'œuvre freudienne, la figure de l'objet dans la forme du moi, il convient de se tourner vers l'œuvre de Mélanie Klein, pour autant qu'elle y trouve à la fois le point de départ légitime de sa recherche et la raison principale du remaniement théorique de fond qu'elle propose s'agissant de la problématique du moi.

\section{Avec Klein. Le moi comme « fonction »}

Par rapport à Freud, dont elle ne cesse de se réclamer comme héritière, on sait que l'originalité principale de Mélanie Klein consiste à marquer, dans le champ de la pratique de la psychanalyse avec de très jeunes enfants, l'intensité de l'activité fantasmatique et la précocité des conflits liés au dualisme pulsionnel de la pulsion de vie et de la pulsion de mort. Vis-à-vis de notre problématique propre, cette expérience de la clinique infantile l'invite à insister sur le rôle fondamental des mécanismes d'introjection et de projection dans la différenciation du moi d'avec le dehors, en s'intéressant d'abord à sa constitution rudimentaire dans les premières étapes de son développement.

De là le premier écart essentiel d'avec Freud : entre un moi décrit comme unifié et bien différencié dans le regard freudien (fût-il dénoncé comme un leurre), et l'état de non-intégration du «premier moi » qu'invite à penser le système kleinien (fût-il posé comme un état « rudimentaire » bientôt dépassé), on comprend l'importance de la distance théorique, tant c'est la nature du moi lui-même qui s'en trouve transformée. C'est que la refonte du moi opérée par Klein est inséparable du mouvement global qu'elle imprime à la théorie psychanalytique - au point que la structure du moi ne soit bientôt plus concevable indépendamment des premières modalités persécutives et dépressives de sa relation à l'objet. 
Il s'agit donc de penser cette originalité de la contribution de Melanie Klein à notre problématique : «bon » ou «mauvais », partiel ou total, externe ou interne, la démultiplication des facettes de l'objet qu'elle décrit complexifie en réalité la généalogie proposée par Freud s'agissant de la différenciation entre le moi et le non-moi, et en radicalise en un sens la porosité métapsychologique.

D’abord parce que le «premier moi » ne se développe, pour Klein, que sur fond de morcellement contre lequel il établit sa cohésion progressivement, le long d'un développement complexe, lié à un travail de l'angoisse, et non pas comme un précipité soudain lié, comme chez Freud, à une subite «nouvelle action psychique » à connotation d'identification imaginaire.

Ensuite, parce que le clivage de l'objet, que Klein postule comme corrélatif de ce morcellement originaire, en une série d'éclats «bons » ou « mauvais » problématise assurément la ligne de partage univoque établie par Freud entre le moi, comme se formant uniformément par l'introjection du bon, et le «non-moi », comme se constituant uniformément dans la projection du mauvais.

Enfin, parce que la mise en évidence de la prolifération d'objets partiels, si elle n'invalide pas tout à fait l'hypothèse freudienne qui considère la naissance du moi comme procédant en son fond de l'identification à un objet perdu, renvoie à une étape ultérieure du développement cette identification sur fond de perte - cette problématique se rapprochant davantage des enjeux de la position dépressive à partir du travail du deuil et des processus d'élaboration de l'objet total. Sur ce point, il convient de penser que la notion $d^{\prime}$ ' «objet interne » fragilise encore les frontières entre moi et objet, et concourt aussi à renforcer la remise en cause du moi vécu comme bien «différencié », en aggravant encore le soupçon psychanalytique porté contre cette fausse évidence. 
L'examen détaillé de chacune de ces problématiques suppose qu'on s'y arrête dans cet ordre, pour envisager successivement 1) le développement du «premier moi » kleinien comme fonction s'épanouissant essentiellement en réaction à l'angoisse ; 2) l'état d'indifférenciation entre le moi et le non-moi que décrivent les fantasmes de la position schizo-paranoïde ; 3) l'acquisition d'une différenciation progressive obtenue dans la position dépressive, sur fond de perte.

Sans artificiellement suivre l'ordonnancement chronologique de l'œuvre kleinienne, ce parcours a le mérite de sérier les points de prolongement et de confirmation, ou, à l'inverse, les points de tension et de réfutation, entre Klein et Freud.

\subsection{Le «premier moi » et l'angoisse, le «premier moi » de l'angoisse}

De même qu'elle avance, en un geste théorique controversé pour l'orthodoxie freudienne, la précocité du développement de l'Edipe et du surmoi, Melanie Klein renouvelle également profondément notre compréhension du développement du moi, en insistant d'abord sur l'importance et la précocité des opérations psychiques garantissant la possibilité pour un tel moi d'assurer sa cohésion. Loin de se contenter, avec Freud, d'une «nouvelle action psychique » qui donnerait subitement au moi sa forme unifiée à partir du morcellement de l'autoérotisme, on sait que la théorisation kleinienne tend au contraire à insister sur l'importance des figures du morcellement que le moi doit surmonter dans sa constitution. Aussi la conception du moi kleinien diffère-t-elle nécessairement de celle de Freud le décrivant comme « diplomate », pour autant qu'il est assimilé, dans les premiers mois de la vie et dans le regard de Klein, plutôt d'abord à un théâtre où se reflète tout un monde intérieur peuplé d'objets persécuteurs ou 
bienveillants - la mise au jour des relations précoces rendant compte d'un certain mode d'appréhension et même d'instauration de l'objet, lequel est indissociable de la prise en compte des ensembles d'angoisses et de défenses qui le définissent.

L'hypothèse fondamentale de Melanie Klein, orientant toute la compréhension de son développement, consiste en effet à assigner au moi comme premier enjeu, vital, d'administrer l'angoisse issue de l'action de la pulsion de mort. Si le système kleinien se réclame sur ce point les avancées de Freud des années 1920 - qui redéfinissent le moi comme étant en grand partie inconscient [9] et siège de l'angoisse [20]-, l'originalité en revanche vient de ce que l'importance de l'angoisse chez le tout jeune enfant force l'hypothèse d'un moi au stade le plus précoce, lequel doit en aiguiller le cours par le biais d'une série de mécanismes de défense.

La force du sadisme et la prégnance de l'angoisse, telles qu'elles se manifestent en effet en séance dans le jeu des enfants, conduit Melanie Klein à postuler que le traitement et les effets de la pulsion de mort constituent l'enjeu primordial du développement psychique. Fidèle à Freud lorsqu'elle adopte l'hypothèse fondamentale du conflit entre pulsion de vie et pulsion de mort, elle s'en démarque toutefois en considérant que «l'angoisse surgit de la pulsion de mort à l'intérieur de l'organisme, qu'elle est sentie comme une peur de l'anéantissement (de la mort) et qu'elle prend la forme d'une peur de persécution » ([21], p. 278). L'originalité kleinienne repose de fait sur ce constat clinique que la crainte d'annihilation, extrêmement active d'emblée, fait subir en permanence au jeune moi des menaces qui ne font qu'augmenter l'angoisse et l'agressivité, le mettant en demeure de déployer un arsenal de défenses pour assurer sa survie [22] ; de là la prégnance du clivage, du déni, de l'idéalisation omnipotente, de la projection et de l'introjection qui se révèlent alors comme autant de modalités par lesquelles le moi tente de défléchir l'instinct de mort vers l'extérieur tout en participant au travail de liaison [23] et aux 
processus d'intégration, le conduisant, progressivement, à différencier le monde interne du monde externe.

Si les tendances œuvrant dans le moi à l'intégration, insufflées par la pulsion de vie ([24], p. 192), sont en jeu dès le début, elles restent encore fragiles et provisoires face aux angoisses générées par la pulsion de mort, angoisses qui ne sont pas seulement liées à un objet, dans la théorie kleinienne, mais littéralement incarnées dans et par l'objet : «La peur de la motion destructrice semble s'attacher tout de suite à un objet - ou plutôt elle est vécue comme la peur d'un objet irrésistible et incontrôlable » ([23], note 2 p. 54). Le moi primitif, alternant entre intégration et désintégration, ne se conçoit ainsi dans le regard kleinien qu'en relation avec une multitude d'objets partiels, chaque expérience vécue correspondant à un objet bon ou mauvais, selon qu'elle est source de satisfaction ou de frustration.

Ce sont alors les défenses déployées par le moi kleinien pour combattre cette angoisse originaire qui détermineront les positions subjectives dites schizo-paranoïde et dépressive, selon la coloration de l'angoisse qui y prévaut. Loin de légitimer trop naïvement l'idée post-kleinienne d'un «noyau psychotique de la personnalité », il convient de s'aviser que c'est bien plutôt «la nature de l'angoisse » que Klein qualifie alors de «psychotique» pour son caractère déstructurant - et non pas les «parties du moi », comme le retiendra une certaine doxa du commentaire critique sur l'héritage de l'œuvre kleinienne [21].

\subsection{Moi schizö̈de, réalité paranoïde : objets partiels et clivage du moi}

À un premier regard critique, le paradoxe de la position schizo-paranoïde, au regard de la différenciation du moi et du non-moi, peut sans doute s'annoncer comme suit: alors même 
qu'une telle «position » tend à montrer comme la bipartition première du sujet et de l'objet est problématique et ne va pas de soi, le vocabulaire notionnel kleinien ne peut s'interdire de recourir massivement à une telle distinction - quitte à faire appel, d'une manière inédite, à l'idée d'« objet interne » et d' « objet externe »-, alimentant la difficulté qu'il prétend résoudre.

C'est sans doute que ces distinctions ne valent qu'en terme d'horizon encore insensé et inaccessible à ce stade pour le premier moi, et ne se légitiment que du lieu où s'écrit la théorie (c'est-à-dire telles que comprises, sur le mode de la reconstruction comme l'a montré Pontalis [25], par un théoricien supposé avoir quant à lui accès à cette différenciation).

À cet égard, les mécanismes d'introjection et de projection eux-mêmes signalent le mieux l'ambiguïté, en ce qu'ils font par définition référence à un intérieur et un extérieur qu'ils sont justement supposés construire - la mise en place de cette dimension étant précisément l'effet progressif de l'élaboration de la position schizo-paranoïde, laquelle est progressivement éclipsée par l'acmé de la position dépressive. Le jeu de ces deux mécanismes dont le moi primitif use de manière ininterrompue marque d'ailleurs la confusion absolue qui règne tout d'abord entre réalité externe et monde interne, les objets extérieurs étant introjectés en même temps que sont projetées sur eux les pulsions libidinales et destructrices : «Ainsi l'image de l'objet, extérieur ou intériorisé, subit dans le psychisme du bébé une distorsion du fait de ses phantasmes, qui sont liés à la projection de ses pulsions sur l'objet. » ([24], p. 189). Sur ce point, on remarque au passage qu'en situant l'incidence du fantasme sur les impressions issues de l'extérieur, subissant inévitablement un remodelage, une déformation du fait des pulsions, cette interaction incessante entre introjection et projection mise en avant par Klein dans le premier fonctionnement psychique complexifie là encore le schéma freudien, tel qu'il l'a décrit en 1915 ([13] voir supra). En effet, là où Freud suggérait qu'une ligne de partage entre stimulus externe et pulsion interne 
s'imposait spontanément au nourrisson à partir d'un «bon critère objectif », soit l'expérience de pouvoir faire taire le premier et de ne pouvoir échapper à la seconde, on voit que rien n'indique, dans le cadre de la théorie kleinienne, que cette force constante soit localisée dans le corps, mais peut bien au contraire en être expulsée par projection, faisant alors retour d'un dehors-dedans indifférencié sous la figure persécutrice du mauvais objet.

Cela donne à penser comme la délimitation d'un moi et d'un non-moi tout comme l'idée d'intériorité et d'extériorité sont impropres à ordonner le vécu fantasmatique de la position schizo-paranoïde. Si la naissance d'un enfant fait naître l'image d'un enfant expulsé en dehors du corps maternel, la position schizo-paranoïde telle que la dépeint Klein ferait en effet plutôt état d'un nourrisson jeté à l'intérieur du corps maternel, seul décor de ce premier monde peuplé d'objets bons, mauvais, idéaux ou terriblement persécuteurs. Du reste, à cette période, les objets se multiplient à l'infini et constituent plutôt un amas, un conglomérat de tout-moi paradoxalement non intégré et confondu avec le corps de la mère comme seul horizon. Aussi, s’il est possible de dire qu'il y a $d u$ moi dès la naissance pour Melanie Klein, ce n'est cependant pas au sens ultérieur d'une instance différenciée, d'un organe unifié ou d'une membrane établissant une frontière entre le soi et l'objet, entre l'ego et l'alter ego, ou entre l'imaginaire et la réalité. La survie du moi précoce et son devenir sont d'abord et avant tout gouvernés par la tentative de maintenir séparés le «bon sein » et le «mauvais sein », que ceux-ci passent à l'intérieur ou à l'extérieur du moi ; prime donc - avant même toute possibilité de mettre de l'ordre dans le monde selon la ligne du dedans et du dehors - la capacité du moi à organiser la prolifération polymorphe des objets, internes comme externes, selon leur «bonne » ou «mauvaise » intention pour le moi. Et encore ces qualités «bonnes» ou «mauvaises » sont-elles à mettre entre guillemets, si tant est qu'elles ne caractérisent pas même l'objet en propre, dans sa nature, mais 
bien plutôt les projections qui l'habillent et le structurent sur tel ou tel mode, pour le moi, dans sa constitution progressive.

Si la logique première de différenciation s'affirme toujours dans ce clivage manichéen poussé défensivement à son extrémité pour Klein, c'est, comme on sait, qu'elle a pour fonction essentielle de protéger le bon objet, duquel dépend, somme son point focal, la constitution du noyau du moi et sa capacité à s'intégrer : «Ce premier objet "bon" intérieur agit comme un point central dans le moi. Il contrecarre les processus de clivage et de dispersion, il appuie la cohésion et l'intégration, c'est un instrument dans la construction du moi. » ([21], p. 297).

Aussi Melanie Klein s'emploiera-t-elle à distinguer à cet égard le bon objet interne de l'objet idéalisé, quant à lui «corrélatif aux angoisses de persécution, c'est à dire qu'il se constitue comme la contrepartie de l'objet persécuteur et qu'il est tout aussi fantasmatique que lui » ([26], p. 133). La répartition des objets par le clivage révèle donc la solidarité de leur construction, en ce que le degré de dangerosité des mauvais objets déterminera défensivement le degré d'idéalisation du bon objet, entravant ainsi la synthèse à venir du moi. Le clivage de l'objet ne restera d'ailleurs pas sans écho sur la structuration du moi, dans la mesure où ce dernier est « incapable de cliver l'objet - interne et externe - sans qu'un clivage correspondant se produise à l'intérieur de lui-même. C'est pourquoi les phantasmes et les sentiments concernant l'état de l'objet interne influencent fondamentalement la structure du moi. Plus le sadisme prévaut dans le processus d'incorporation de l'objet, plus l'objet est senti comme fragmenté, et plus le moi est en danger d'être clivé selon les fragments de l'objet intériorisé » ([21], p. 280). Le moi, tout à la fois clivant et clivé, se déchire donc de l'intérieur de par sa propre agressivité, une destructivité interne difficile à projeter entièrement vers l'extérieur au départ.

Entendons que, pour Klein, non seulement l'objet interne ne recouvre pas si simplement 
le moi, pas plus que l'objet externe le ferait avec le monde extérieur, mais qu'il n'y a de surcroît pas de figure du moi possible dans l'approche kleinienne sans articulation de la logique de ce clivage de l'objet. Si le moi est le lieu où siègent angoisses et défenses, incarnées dans les objets, il est aussi lui-même constitué par l'objet auquel il est en un sens «asservi » : ce qui revient à penser que ce n'est pas le moi qui créé ses objets dans un rapport au monde morcelé, comme une certaine doxa psychanalytique pourrait le laisser entendre, mais plutôt bel et bien l'objet qui délimite les frontières du moi, qui n'en est à l'occasion que la « coquille » $\mathbf{1 0}$.

Mais parvenu à ce point, on s'avise pourquoi, à une perspective partant de la bipolarité du moi et de l'objet, la théorie kleinienne substitue une perspective donnant plutôt à penser la polarité de l'objet dans le moi. Et c'est pourquoi en articulant moi, clivage, introjection et projection, objets internes et externes, Klein déstabilise si considérablement la théorie freudienne qui faisait coïncider le moi avec l'introjection du bon et le non-moi avec la projection du mauvais, en n'hésitant pas, elle, à évoquer la possible projection de bonnes parties du moi afin de les préserver des attaques des mauvais objets internes, tout comme la possible introjection d'objets mauvais dans le but omnipotent de mieux les contrôler.

La pointe de cette complexification réside dans la mise à découvert de l'identification projective, cette projection dans l'objet de parties clivées du moi (bonnes ou mauvaises) - parties expulsées auxquelles le moi demeure cependant identifié. On ne peut que souligner la porosité de conformation de «l'intérieur » et de «l'extérieur », pour le sujet kleinien, spécialement mise en valeur par ce mécanisme où la projection des parties clivées du moi se fait non pas tellement sur mais dans l’objet (« des parties clivées du moi sont aussi projetées sur la mère ou, pour mieux

10 « Il peut en résulter que le moi se sente entièrement asservi à son objet interne et entièrement dépendant de lui, comme s'il n'était pour lui qu'une coquille. On trouve donc, en même temps qu'un objet idéalisé non assimilé, le sentiment que le moi n'a ni vie ni valeur propres. » ([21], p. 283-284). 
dire, dans la mère. » [21], p. 282), ce qui, de ce fait, «permet de confondre le soi 11 et l'objet qui vient à le représenter. » ([27], p. 35).

Si cette série de mécanismes schizoïdes mise en place par le moi n’a rien de pathologique en soi, dans la mesure où ils visent à se défendre de l'angoisse de persécution, c'est leur usage excessif et rigide qui, en revanche, entrave l'élaboration de la position schizo-paranoïde, en ce qu'il appauvrit le moi et grève ses tentatives d'intégration, renforçant encore la dislocation du moi et la porosité des limites du moi et du non moi.

Mais en ces premiers temps du développement, on vérifie que la frustration, le manque et l'absence n'imposent rien de l'épreuve de réalité ou d'un champ hors-moi puisqu'ils s'interprètent, tout au contraire, comme des attaques convoquant un trop de présence de l'objet mauvais et persécuteur. Dans les premières expériences que l'enfant fait du manque, l'absence n'est jamais que la présence d'une persécution, pour le regard kleinien. C'est que la délimitation d'une frontière permettant cette sorte d'absentification de l'objet implique, dans la théorie kleinienne, qu'entre en jeu la dimension de la perte comme condition essentielle, en conséquence des processus d'intégration du moi et de l'objet.

\subsection{Le moi dépressif : objet total et consistance du moi}

Les facteurs déterminant la mise en place des limites entre moi et non-moi relèvent chez Melanie Klein de conditions essentiellement internes et fantasmatiques, fonction de la manière dont le conflit pulsionnel peut être traité par le moi. Là est la fonction du symbole : « À mesure

11 Probablement comme conséquence du débat anglophone pendant les dernières années d'exercice de Melanie Klein, le mot self a pris une place de plus en plus grande dans son œuvre. Le self ne recouvre pas seulement le moi mais implique de manière plus large toutes les instances psychiques. Comme on sait, Winnicott s'est quant à lui très tôt attaché à cette notion, voir infra. 
que le moi se développe, une relation authentique à la réalité s'établit progressivement à partir de cette réalité irréelle. [...] Il faut une quantité suffisante d'angoisse pour fournir une base à une abondante formation de symboles et de fantasmes. » [28], p. 265). C'est ici que jouent les effets de la pulsion de vie, qui s'expriment d'emblée par les tendances à l'intégration, mises en péril par la désintégration impulsée par la pulsion de mort : entendons que la maturation du moi et sa capacité à administrer l'angoisse vont dépendre de la manière dont le moi établit et installe à l'intérieur de lui le bon objet avec suffisamment de confiance et de sécurité : «le bon objet intériorisé vient former le noyau du moi autour duquel il s'étend et se développe. Car, lorsque le moi est soutenu par le bon objet intériorisé, il est plus à même de maitriser l'angoisse et de préserver la vie en liant avec la libido certaines parties de l'instinct de mort agissant à l'intérieur. » ([23], p. 55)

L'ancrage du bon objet intériorisé a, dans sa solidité, une fonction de cohésion centrifuge pour le moi, en permettant l'élaboration de l'angoisse de persécution - au sens d'une diminution de la destructivité aussi bien que des mécanismes qui visaient à s'en défendre -, ce qui favorise ainsi le déploiement des processus d'intégration. Ceux-ci touchent au sentiment d'unification du moi et, dans le même mouvement, à la possibilité de synthétiser les objets partiels en un objet total, en un processus qui ne s'opère pas sans douleur et sans l'émergence d'un nouveau danger qui caractérisera l'angoisse dépressive. Car à découvrir que le bon sein et le mauvais sein ne forment qu'une seule et même personne - la mère -, à rapprocher ainsi les aspects contrastés des objets partiels pour former un objet total à la fois aimé et haï, et, bien que le pouvoir des pulsions destructrices ait diminué, «ces pulsions sont senties comme un grand danger pour l'objet aimé, qui est maintenant perçu comme une personne » ([24], p. 200]. Le bon objet se retrouve alors mis en péril par la haine du sujet, en danger de mort, menacé d'être irrémédiablement perdu, 
littéralement détruit par les pulsions agressives jusqu'ici projetées sur le mauvais objet soigneusement tenu séparé du bon par le clivage. Le danger couru par l'objet aimé constitue non seulement un horizon angoissant faisant apparaître préoccupation et souci à l'égard de l'objet dans le but de le préserver et de le maintenir en vie, mais également une perte effective. L'objet total n'est en effet pas à considérer comme la somme exacte des objets partiels réunis, il y a un rebut, un reste dans cette opération en ce qu'il implique chez Klein la perte et le deuil de l'objet idéal. Précision décisive : l'objet total, noyau du moi chez Klein, n'est pas un objet complet, il est entamé - ce pourquoi cette perte à partir de laquelle le moi kleinien va enfin trouver une cohésion rejoint l'hypothèse freudienne de la formation du moi sur fond de perte (de l'objet).

C'est la raison essentielle pour laquelle la formation de l'objet total, permettant une véritable réorganisation du rapport du moi au monde, ne peut s'effectuer que sur fond de deuil, dans un climat dépressif, culpabilisateur et nostalgique, que signale à l'occasion cliniquement le franchissement de l'enfant d'un seuil dépressif, dans son rapport à l'objet.

La perte en jeu dans la construction de l'objet total se fait ainsi condition essentielle de l'instauration des frontières entre le moi et le non-moi, ce dernier champ se référant alors à la réalité extérieure dans laquelle s'insère l'objet externe, enfin différencié de l'objet interne. En d'autres termes, l'émergence du champ du non-moi à l'orée de la position dépressive - et en dépit des défenses mobilisées par l'angoisse de perte - implique un remodelage du moi du côté de l'unification et de la perpétuation des processus d'intégration des objets internes, d'une part, corrélatives de la délimitation de ses limites d'avec l'extérieur, d'autre part. La maturation du moi suppose en effet la capacité graduelle du moi à distinguer «la frustration imposée de l'extérieur de ses dangers internes phantasmatiques » ([24], p. 203), en lieu et place de l'objet persécuteur comme figure unique et condensatrice. Cette distinction de ce qui provient du dedans 
et du dehors va se doubler d'une concordance entre monde intérieur et monde extérieur, d'un rapprochement plus réaliste entre objets internes et externes de par la diminution des aspects fantasmatiques terrifiants ou idéaux grâce à l'adaptation croissante à la réalité ([24], p. 200 et 203). Melanie Klein formulera ainsi que «si l'angoisse persécutive n'est pas excessive, et si la capacité d'amour est assez forte, le moi se rend de plus en plus compte de sa réalité psychique et ressent de plus en plus que c'est ses propres motions destructrices qui contribuent à abimer ses objets. Ainsi les objets blessés, qui étaient ressentis comme mauvais, se sont améliorés dans l'esprit de l'enfant et se rapprochent plus des parents réels; le moi développe peu à peu sa fonction essentielle de traitement du monde externe. » ([24] p. 59).

Dans sa description de la position dépressive, Klein dira que « le moi semble arrivé à un carrefour d'où rayonnent plusieurs routes ; du choix d'une de ses routes dépend la constitution psychique toute entière » ([29], p. 340). La position dépressive n'est pas une finalité en tant que telle mais un moment crucial pour la structuration psychique : ce qui y est décisif est bien le traitement de la perte et donc le deuil associé à celle-ci [30].

En effet, atteindre la position dépressive sans pouvoir l'élaborer implique corrélativement que le bon objet ne soit pas installé à l'intérieur du moi de façon suffisamment sécurisée, et que l'objet ne se constitue pas comme total - d'où l'éventuelle régression à un mode de défense typique de la position schizo-paranoïde, ou un recours massif aux défenses maniaques pour parvenir à supporter l'angoisse dépressive. C'est donc dans la possible introjection de l'objet comme un tout incomplet (réunissant ses aspects haïs et aimés) que se trouve le choix des névroses ou des psychoses.

Or, dans cette description génétique d'ensemble, on vérifie que le développement du moi dépend de facteurs que Klein postule comme étant pour une grande part innés [31]. La part 
dévolue à l'influence environnementale dans la constitution du moi - et ceci forme un point de dissension majeur d'avec Winnicott - occupe une place ambiguë chez Klein, en ce que son évocation tend toujours à résonner comme une concession, sans jamais être tout à fait niée pour autant 12. Ce point d'ambiguïté s'éclaire à considérer précisément qu'une telle différenciation entre intérieur et extérieur, dedans et dehors, moi et non-moi n'opère pas dans les premiers temps de la position schizo-paranoïde ; mais laisse dans l'ombre - problème trop vite résolu par le recours aux «facteurs constitutionnels »- les motifs qui rendent compte des disparités dans ladite force du moi. Le cachet typiquement kleinien n'en restera pas moins celui d'avoir orienté avec force la psychanalyse de l'enfant en en réglant les principes directeurs sur le monde fantasmatique de l'enfant d'abord - ce qui ne manque pas de laisser intouchée, parfois, la question de la mère et des effets de sa propre ambivalence. Mais c'est précisément ce qui engage à prendre acte, à l'opposé, de l'incidence de l'environnement dans la constitution du moi.

\section{Avec Winnicott. Là où était du ça, du self doit advenir ?}

Par rapport à l'ego kleinien, la reprise par Winnicott du Ich freudien se singularise essentiellement par trois aspects. Premièrement, Winnicott entend refonder la question de la constitution du moi de l'infans en mettant au premier plan la fonction de l'environnement lors des relations précoces - en déplaçant donc l'accent que Freud avait mis, de manière inaugurale pour la pensée psychanalytique, sur la constitution du moi dans son rapport au ça. Ceci le conduit, deuxièmement, à formuler des hypothèses originales concernant les rapports du moi

12 «La mesure dans laquelle la force du moi peut être maintenue et accrue est en partie affectée par des facteurs externes, en particulier l'attitude de la mère à l'égard du nourrisson » [23]. 
avec la réalité - l'invention de l'espace transitionnel et le thème de la «survie de l'objet » constituant des innovations inédites au sein du champ analytique. Enfin, son originalité consiste à promouvoir la notion de self comme nouvelle réinterprétation du Ich freudien, distincte aussi bien de la notion d'ego (telle que reprise par Klein, mais, également, par l'ego-psychology) que des concepts de moi et de sujet (proposés par Lacan). Ce faisant, il instaure un nouveau couple propre à structurer la pensée psychanalytique : le vrai et le faux self - déplaçant, ce faisant, les fondements de l'édifice psychanalytique tout autant que les frontières permettant de penser le moi.

\subsection{Rôle de l'environnement dans la constitution du moi}

Afin de penser la constitution du moi 13 , l'originalité de Winnicott consiste à mettre l'accent sur le rôle de l'environnement. «Ce n'est pas l'individu qui est la cellule, mais une structure (set-up) constituée par l'environnement et l'individu», nous dit-il. Et il ajoute «Le centre de gravité de l'être ne se constitue pas à partir de l'individu : il se trouve dans la structure environnement-individu. Une bonne technique de soins appropriés (good enough) à l'enfant [...] se substituera graduellement à la coquille, et le noyau - qui pour nous, n'a pas cessé de ressembler à un petit enfant d'homme - pourra commencer à devenir un individu. » ([34], p. 201).

13 Précisons ici que les termes winnicottiens pour désigner le moi sont nombreux : si l'opposition me/not-me est commode pour sa clarté et si l'usage du $I(« I \mathrm{am} »)$ est propre à Winnicott, ces usages ne doivent pas cacher sa relative indécision quant à l'usage des termes ego et self. $\mathrm{Si}$, en certaines occurrences, les deux termes sont interchangeables (notamment au début de son œuvre comme en 1956 ([32], p.174), ils sont, au cours des années 1960, de plus en plus fermement distingués, vraisemblablement à mesure qu'est élaborée la notion de self, et ce, au point d'être radicalement distingués en 1970 : «le self... n'est pas l'ego » [33]. Schématiquement disons que l'ego sera de plus en plus appelé à désigner une fonction de synthèse plutôt qu'à entendre comme instance de la deuxième topique. Le self, innovation théorique de Winnicott, sera amené à désigner ce cœur constitutif de l'irréductible singularité de chacun - par opposition au faux self. 
Cette mise en exergue, fruit de ses observations des relations mère-bébé en tant que pédiatre, s'inscrit en réaction à toute fiction théorique qui envisage la constitution du moi et du non moi selon la dialectique de la pulsion et de l'objet - qu'il s'agisse des théorisations kleiniennes qui surestiment, à ses yeux, le pôle pulsionnel au détriment de la manière dont il est pris soin de l'infans par l'environnement ou qu'il s'agisse de la classique version freudienne mettant en avant le rôle de la déception primordiale dans l'épreuve de réalité.

En étant plus nuancé, il est peut-être plus juste de dire que la grande thématique winnicottienne de l'environnement vient mettre l'accent sur un aspect effleuré, mais jusque-là demeuré largement négligé dans la théorie psychanalytique. En effet, Klein elle-même s'était trouvée contrainte de mentionner l'impact des «facteurs externes » dans sa version du moi précoce ([35], p. 99) - et ce, même si sa pensée demeure fondamentalement innéiste et constitutionnaliste. Davantage, Freud lui-même avait déjà suggéré l'importance des « soins de la mère » pour rendre vraisemblable la «fiction » de son schéma explicatif de l'appareil psychique en 1911, selon la métaphore de l'œuf «pour lequel les soins maternels se restreignent à l'apport de chaleur» ([12], p. 14-15). Véritable héritage ou vrai désir d'asseoir la légitimité de ses recherches sur l'environnement (notamment contre Klein), Winnicott ira d'ailleurs jusqu'à se demander s'il n'a pas été « influencé, sans le savoir, par cette note de Freud » ([36], p. 361).

Car, pour Winnicott, dans cette fiction théorique de la constitution du moi, il s'agit de comprendre que les termes du problème se trouvent d'emblée mal posés. Envisager les rapports du bébé et de la réalité constitue une erreur de perspective. En effet, son intuition fondamentale, formulée «avec fougue » dès le début des années 40 (et à son propre étonnement, au moment même où il était en train de la proférer), consiste à considérer que «there is no such thing as a baby » ([34], p. 200) et ([36], p. 361). Un bébé, tout seul, ça n'existe pas - en tout cas, pas sans 
les soins qu'un «autre » lui prodigue. Par conséquent, il n'existe tout simplement pas d'entité indépendante, animée de pulsions, cherchant la satisfaction et susceptible d'expérimenter quelque déception que ce soit en provenance de la réalité : le bébé n’est pas « une personne qui a faim et dont les besoins instinctuels peuvent être satisfaits ou bien frustrés » ([37], p. 11).

L'infans, à l'origine dans un état de dépendance absolue, est, au contraire, indissociable de la dyade qu'il constitue avec la mère ou son représentant ([34], p. 201) et c'est à partir de celle-ci qu'il convient de repenser la constitution du moi. Dans ce premier temps, aucun moi de l'enfant n'est intégré (constat que Klein reprend à Winnicott dès 1946 ([21], p. 278]) : «Il est permis de présumer que, au début théorique, la personnalité est non intégrée » ([38], p. 63).

À partir de là, la thèse de Winnicott est bien connue : seule une mère «suffisamment bonne» ou «suffisante», traductions imparfaites de «good enough», est susceptible de permettre l'émergence du moi, par sa «dévotion» au début totale (permise par cette folie passagère, ou «état psychiatrique » qu'est la «préoccupation maternelle primaire » ([32], p. 287]. Cette adaptation presque absolue de l'environnement déborde largement le fait de satisfaire les «besoins » de l'infans (le fait de nourrir le bébé par exemple). Winnicott ne cesse, en effet, de remettre en question la conception, qu'il considère comme réductrice, de l'enfant comme être pulsionnel, mû par le ça, sujet à la satisfaction ou la frustration - tant et si bien, d'ailleurs, qu'une gratification purement pulsionnelle peut ne pas être «satisfaisante » pour le petit enfant ([38], p. 68), voire se révéler «séductrice » et «traumatique » pour le moi naissant ([39] p. 117).

Comme, à ce stade, l'infans est « un être immature », tout le temps au bord d'angoisses impensables (unthinkable) - se morceler, ne pas cesser de tomber, ne pas avoir de relation avec son corps et ne pas avoir d'orientation - le rôle de l'environnement consiste à assurer une 
fonction de «coquille» protectrice visant à contenir celles-ci [32] et à rendre possible le développement du «noyau » du moi encore non intégré - ce «noyau » qui, jusque-là, «pour nous, n'a pas cessé de ressembler à un petit enfant d'homme» ([34], p. 201).

Cet ego-coverage ou « soutien du moi » de la part de l'environnement consiste à favoriser plusieurs processus. Le processus d'intégration (1), schématiquement, désigne le travail de synthèse ; il instaure l'unité et la continuité du moi - à l'origine de «l'instauration de l'état correspondant à “'Je suis” » ([37], p. 16). Il émerge du holding, littéralement portage du bébé (au sens physique, mais également psychique). La personnalisation (2) désigne l'union du moi et du corps ou cohésion psychosomatique, la peau venant incarner, physiquement, la membrane frontière qui permet de séparer le moi du non moi. Celle-ci suppose un handling adéquat, c'est-àdire une manipulation du corps du bébé qui permet l'enracinement de la psyche dans le corps (ou constitution du «psyche-soma »). Enfin, la possibilité d'entrer en relation avec les objets (object relating) (3) est possible à condition que l'environnement présente les objets à l'enfant (object presenting) au moment opportun, c'est-à-dire au moment où celui-ci est susceptible de le chercher et donc de le créer - ce qui inaugure la grande thématique winnicottienne de l'espace d'illusion.

Avant d'en arriver là, soulignons que les défaillances de l'environnement constituent donc des obstacles majeurs au développement du moi. Premièrement, elles laissent le nourrisson sans protection face aux angoisses impensables. Dans ce cas, celui-ci en est réduit à se défendre par la « désintégration » (à distinguer de la «non intégration », état originel d'où émergent la créativité et le self). Deuxièmement, face à un environnement qui ne s'adapte pas mais exerce une «pression » trop forte sur le moi [40], ne reste que la solution de l'élaboration d'un faux self (voir infra). 
Pour conclure cette partie, force est de constater que l'originalité de Winnicott, voire son hétérodoxie vis-à-vis de la découverte freudienne (et tout autant vis-à-vis de sa reprise kleinienne), consiste à penser l'émergence du moi en relation avec l'environnement plutôt que de l'envisager dans ses relations avec le pulsionnel ou le ça.

D'ailleurs, à ses yeux, c'est le moi qui est premier - «the start is when the ego starts »et «le ça n'existe pas avant le moi » ([37], p. 9). Chez Winnicott, c'est l'environnement qui prime et qui permet au moi de l'infans d'intégrer, dans un deuxième temps, la part pulsionnelle qui vient, en retour, renforcer le moi. Cette relégation dans les limbes de la part pulsionnelle dans la fiction

de l'édification du moi, ou, plus exactement, cette possibilité «de ne pas en tenir compte » $\mathbf{1 4}$, révèlent le déplacement que Winnicott fait subir à la théorie analytique et, ce faisant, à la pratique de la cure. Deux remarques gagnent ici à être faites pour envisager la portée de cette proposition théorique.

Rappelons (1) que ce déplacement, pour être pleinement saisi, doit être mis en rapport avec la rencontre clinique de Winnicott avec les patients non névrosés (pour lesquels le conflit moi-refoulé ne serait pas sur le devant de la scène). Ensuite (2), soulignons qu'en reléguant au deuxième plan le conflit entre le moi et la pulsion dans l'inauguration du moi - thème inaugural de la découverte freudienne - ou en d'autres termes, en déplaçant les frontières du moi proposées par Freud vis-à-vis du refoulé, Winnicott vient sans doute bouleverser, peut-être malgré lui, les fondements mêmes de la théorie psychanalytique et en reconfigurer l'espace. En anticipant sur la suite, disons qu'il est sans doute lourd de conséquences qu'il déplace la frontière moi/refoulé au

14 Tant que la vie instinctuelle « existe en dehors du fonctionnement du moi » - la pulsion paraît aussi étrangère au bébé qu'un «coup de tonnerre »-1'on peut, nous dit Winnicott, «ne pas en tenir compte» ([37], p. 9), nous soulignons). 
profit d'une nouvelle frontière vrai/faux self. Quant aux frontières moi/environnement good enough, leur non existence, dans un premier temps, permet d'offrir, par procuration, au moi émergeant, la coquille de protection dont il a besoin pour constituer, dans un deuxième temps, ses propres frontières. En d'autres termes, le moi émergeant trouve un étayage sur les frontières que lui offre l'environnement suffisamment bon pour prendre corps et constituer sa propre membrane frontière. Il est à noter que la conflictualité ou les querelles de frontières entre le moi et l'environnement ne surgissent que lorsque l'environnement, pas suffisamment bon, empiète, telle une puissance impérialiste, sur le moi encore non intégré de l'infans.

\subsection{Le moi, l'illusion et la réalité}

Dans ce cadre de pensée, qu'en est-il de l'instauration de l'épreuve de réalité et de la distinction moi/non-moi (me/not-me) ? La proposition de Winnicott se révèle ici, comme souvent, paradoxale : l'accès à la réalité n'est possible, à ses yeux, que sur fond d'illusion ou, pour reprendre sa métaphore, que si l'infans a pu d'abord faire l'expérience d'en avoir été, tel Dieu, le créateur [41]. Non content de mettre en question la conception de l'infans comme être de pulsions, Winnicott, introduisant «l'aire transitionnelle », à la fois moi et non moi, entend repenser la classique opposition entre réalité interne et réalité externe ainsi que le mythe freudien de la constitution de la réalité.

Voici quelles sont les étapes de ce processus. Dans un premier temps, un environnement suffisamment bon, nous dit Winnicott, doit pouvoir s'adapter aux exigences, au début, absolues de l'enfant et ce, afin de lui permettre d'expérimenter l'illusion d'omnipotence. «La mère place le sein réel juste là où l'enfant est prêt à le créer, et au bon moment » nous dit Winnicott dans son 
célèbre article sur les objets transitionnels ([42], p. 21). Dans ce cas, la présence du sein ne satisfait pas seulement la faim, elle donne l'illusion au bébé d'être tout puissant et lui permet d'expérimenter sa créativité primaire. Le paradoxe, qui a vocation à ne pas être résolu, c'est que si l'objet doit tout d'abord être créé par l'infans avant d'être trouvé, l'objet doit pourtant être trouvé pour pouvoir être créé. À ce stade, bien sûr, l'objet est un phénomène subjectif, sous contrôle magique et non un objet de la réalité objective.

Dans des conditions favorables, des défaillances mesurées, après un moment d'adaptation presque parfaite de la mère à l'enfant, permettent une sortie progressive de l'omnipotence et la constitution d'une aire d'illusion, se situant dans cet espace paradoxal où se chevauchent l'espace interne (l'aire de la créativité primaire) et l'espace externe (l'aire de la perception objective basée sur l'épreuve de réalité). C'est dans cette aire intermédiaire, aux alentours «du quatrième, sixième, huitième au douzième mois », qu'est susceptible d'être trouvé/créé cet objet paradoxal, l'objet transitionnel. Prenant la place du sein (ou objet de la première relation), il permet de parer à l'angoisse de type dépressif, indissociable de la phase de désillusion et de sevrage (aussi le petit enfant ne peut-il l'inventer qu'à condition que l'objet interne soit «vivant, réel et suffisamment bon (pas trop persécuteur) »-ce qui, bien sûr, dépend « de l'existence, du caractère vivant (aliveness) et du comportement de l'objet externe » ([42], p. 18-19) et non de sa constitution interne comme le suppose Klein.

L'objet transitionnel, première «possession non-moi », est distinct d'un «objet subjectif » (ou objet interne au sens kleinien) ([42], p.18-19) et, à ce titre, il constitue une étape dans la progressive sortie du solipsisme. Cela n'en fait pas pour autant, pour le nourrisson, un objet externe : s'il peut exercer un contrôle sur lui (ce qui n'est pas le cas avec la mère), ce contrôle n'est pas pour autant de type magique et une certaine annulation de l'omnipotence est 
déjà en jeu. Cette perte se trouve contrebalancée par la matérialité de l'objet transitionnel, qualité qui lui permet de survivre à l'amour instinctuel et à la haine, voire aux traitements «sans égards » de l'infans - survie dont est incapable l'objet halluciné. Comme le résume Winnicott, l'objet transitionnel, qui précède l'établissement de l'épreuve de réalité, est un objet paradoxal : «On peut dire à propos de l'objet transitionnel, qu'il y a là un accord entre nous et le bébé comme quoi nous ne poserons jamais la question : “Cette chose, l'as-tu conçue ou l'a-t-elle été présentée du dehors ?" L’important est qu'aucune prise de décision n'est attendue sur ce point. La question elle-même n'a pas à être formulée. » ([42], p. 23).

L'objet transitionnel ménage donc une transition dans l'accès à la réalité, mais il en précède l'établissement. À partir de là, comment donc penser la sortie du solipsisme ? Dans une première partie de son œuvre, Winnicott se range partiellement à la version freudienne : «Si tout se passe bien, l'enfant peut effectivement bénéficier de l'expérience de la frustration, car une adaptation incomplète au besoin rend les objets réels, c'est-à-dire aussi bien haïs qu'aimés. » ([42], p. 20).

Néanmoins, ses ultimes développements théoriques tendent à renverser la perspective. Désormais, la constitution de la réalité ne résulte pas tant d'une «déception» que de la « survie » de l'objet et de la joie qui en accompagne la découverte. Si ce thème est présent en filigranes dès 1951 (puisque l'une des vertus de l'objet transitionnel est de «survivre », ce que sous-entend la formule «si tout se passe bien»), voire dès 1945 (comme l'importance de la répétition et de la «monotonie» des gestes maternels le suggère ([38], note 1 p. 67), la proposition selon laquelle c'est la destructivité, et par suite la survie de l'objet, qui fabriquent la réalité (et non l'absence de celui-ci au moment où l'infans est prêt à le créer) émerge clairement en 1963 à la suite d'un rêve théorique décisif ([43], p. 243). Dans «L'usage de l'objet et le mode 
de relation à l'objet au travers des identifications », il théorise ce point et propose la séquence suivante : «1) le sujet se relie [relates] à l'objet. 2) L'objet est en train d'être trouvé au lieu d'être placé dans le monde par le sujet. 3) Le sujet détruit l'objet. 4) L'objet survit à la destruction. 5) Le sujet peut utiliser l'objet. » ([44], p. 242). La sortie de l'objet hors de l'aire d'omnipotence s'opère selon une double logique du fait que «le sujet détruit l'objet parce que l'objet est situé en dehors de l'aire de son contrôle omnipotent » et, en sens inverse, du fait «que c'est la destruction de l'objet qui place celui-ci en dehors de l'aire du contrôle omnipotent du sujet. » ([44], p. 236)

Notons que cette agressivité primaire (à laquelle Winnicott donnera plusieurs noms : primary agression, ruthlesness, fait d'être sans pitié [38] ou destruction) n'est pas à entendre au sens kleinien de la pulsion de mort, mais comme traitement «cavalier » de l'objet ([44], p. 235), mêlant amour et haine, sans considération ni pitié, mais également sans colère ni envie. La « survie » de l'objet désigne le fait que le sein est toujours là, vivant et qu'il n'applique pas de « représailles » (à l'inverse d'une mère «non suffisamment bonne » qui, trop atteinte par l'agressivité de son bébé, se sentirait menacée voire persécutée par les exigences de celui-ci) ([44], p. 235).

L'étape de la naissance de l'objet est donc contemporaine de la naissance du fantasme : l'agressivité primaire (destruction dit-il en 1969) n'ayant pas eu raison de l'objet, elle s'instaure comme «fantasmatique». Fantasmatiquement parlant, l'objet (en tant que non-moi) est d'ailleurs, selon lui, « toujours en train d'être détruit » ([44], p. 242)

Elle inaugure également la possibilité de l'utilisation de l'objet, distincte de l'objectrelating (fait d'être en relation avec l'objet-subjectif). Cette «utilisation» qui doit être fermement distinguée de toute «exploitation » comporte un sens positif ([44], p. 241), au point 
que la fonction de l'analyste consiste, comme l'objet primordial, à « rester vivant » dans la cure, sans représailles et à se laisser « utiliser » (indication clinique qui s'applique essentiellement ou en tout cas électivement aux «psychoses structurées sur un mode pseudo-névrotique »).

Ces deux mythes théoriques de la constitution de la réalité («déception » par l'objet versus « survie » de l'objet) sont-ils incompatibles comme cela semble être le cas à première vue ? Ce n'est sans doute pas si simple. Premièrement, il semble que Winnicott ne conteste pas tant la version freudienne qu'il n'insiste sur ce qui rend possible cette étape : si seule la déception était expérimentée, elle ne pourrait conduire à aucune installation du principe de réalité, mais seulement à la soumission à une réalité vécue comme implacable, voire à la mort psychique et physique. Deuxièmement, dans les deux cas, la constitution de l'objet non-moi comporte, malgré la contradiction apparente, une structure commune. Que ce soit par l'expérience de la frustration (en raison de l'absence de l'objet) ou par l'expérience de la surprise que suscite la survie de l'objet (en dépit des attaques dont celui-ci a été la cible), la réalité vient démentir l'omnipotence fantasmatique et inaugurer une différenciation entre monde interne et monde externe. Néanmoins, là où Freud insiste sur la déception et Klein sur l'angoisse que suscite la rencontre avec l'objet de la réalité (notamment de par ses disparitions), Winnicott, comme toujours, préfère insister sur la « joie », ici celle de découvrir que ce dernier «survit» ([44], p. 241).

\subsection{Faux self et vrai self}

L'ultime apport de Winnicott à la question du moi concerne la notion de self. De même que Locke, en réponse aux théories continentales de l'ego, notamment cartésien, en avait déjà établi la consistance pour la philosophie anglo-saxonne, l'introduction de ce terme en 
psychanalyse pourrait apparaître comme une simple «naturalisation » du Ich freudien dans la langue anglaise (qui ne comportait, pour Klein, germanophone, aucune nécessité). Néanmoins la mise en exergue de ce terme, aux dépends de l'ego, terme savant et médicalisant d'origine latine choisi par Jones (et repris par Strachey) pour traduire Freud, dépasse le simple choix linguistique. Passer du Ich à l'ego pour en arriver au self implique un profond remaniement théorique 15. À cet égard, on constate d'ailleurs que les principaux courants psychanalytiques (à l'exception de Melanie Klein peut-être) en viendront tous à promouvoir, de manière extrêmement révélatrice quant à leur orientation, des termes nouveaux pour traduire le Ich freudien. Si l'ego-psychology fonde sa théorie sur l' «ego », Lacan repensera le Ich de Freud en proposant la distinction entre le «moi » et le «sujet» et Winnicott lui substitue le self.

Mais avant d'en arriver là, il est important de noter que la nécessité de l'introduction des notions de vrai et faux self est, à nouveau, fondamentalement clinique - fruit du travail de Winnicott avec les psychoses « dépourvues des manifestations psychiatriques classiques » [45] souvent rapprochées des personnalités « as if » de H. Deutsch.

De manière un peu schématique, on pourrait soutenir qu'en introduisant le self, Winnicott remplace le conflit moi/refoulé (ou moi/ça/surmoi) par un nouveau couple d'opposés au cœur de la psyché : le vrai et faux self. Si l'article de 1960 «La distorsion du moi en fonction du vrai et du faux self » est souvent pris comme repère pour dater l'instauration de ce couple notionnel, les termes apparaissent en réalité bien avant, au moins depuis les années 1950 ([46] et [45]).

Comme nous l'avons déjà annoncé, cette dialectique entre vrai et faux self trouve son origine dans les relations primaires de l'infans avec l'environnement [39]. L'instauration du faux 
self résulte, selon Winnicott, des défaillances de l'environnement lors des interactions précoces. Sans la protection offerte par un environnement suffisamment bon permettant au vrai self de prendre corps, l'infans se voit contraint de se soumettre aux contraintes extérieures, c'est-à-dire de développer un faux self au détriment du vrai self qui s'atrophie et se coupe de toute relation au monde. Défense élaborée contre l'empiètement (impingement) de l'environnement, le faux self désigne un fonctionnement non pas actif, mais réactif, parfois extrêmement sophistiqué (par son recours à l'intellect, par exemple), mais fondamentalement soumis aux exigences extérieures et, par suite, indissociable d'un sentiment de futilité et de vacuité. Il ne se constitue pourtant pas seulement, comme on le retient souvent, de manière antagoniste au vrai self. Sa constitution vise, en effet, également à sauvegarder celui-ci et notamment à le préserver du risque d'annihilation dont l'environnement le menace. D'ailleurs, tout individu, tôt ou tard confronté à une relative inadéquation de la réalité, forge un faux self. Mais il en existe plusieurs types que Winnicott propose de classer selon un continuum [39]. À une extrémité, un faux self totalement coupé du vrai self et fonctionnant dans la pleine ignorance de celui-ci (en raison d'un clivage), à l'autre extrémité, un faux self protecteur qui joue le rôle d'interface avec la réalité et se charge de faire les compromis que celle-ci réclame afin de mettre à l'abri le vrai self - en bonne intelligence avec celui-ci.

Le vrai self, lui, demeure une notion difficilement saisissable. Winnicott avoue lui-même ne pas savoir exactement ce qu'il désigne [33]. L'expression selon laquelle « le geste spontané, c'est le vrai self en action » [39] permet, sans doute, d'en saisir au plus près la singularité. Noué au soma, sans pour autant être de nature strictement pulsionnelle, le self est porteur de la singularité irréductible de l'individu. Dans le cas favorable où il est accueilli/reflété par la good enough mother, il permet d'éprouver un sentiment de continuité d'existence et de se sentir réel, 
vivant et créatif 16. Dans l'étonnant article de 1963 «De la communication et de la noncommunication suivi d'une étude de certains contraires » [47], cette dimension vitaliste se noue à une dimension «sacrée »- au point que l'expérience du «Je suis » (I am) se trouve rapprochée du tétragramme divin «Je suis celui qui suis » ([48], p.112). Non sans quelque accent mystique, le self y est présenté comme ne communiquant pas avec les objets de la réalité, demeurant à jamais seul, secret et silencieux. À son sujet, Winnicott déclare paradoxalement qu'émane de lui une «communication » qui n'est pas «non verbale » mais qui, bien plutôt, « comme la musique des sphères, est absolument personnelle » [47].

Cette définition, bien sûr, oriente la cure - qui se pense, à partir de là, comme recherche du self [49] - l'innovation de Winnicott consistant à affirmer que pour accéder à celui-ci (et donc à la créativité), le patient n'a pas besoin d'interprétations, mais d'une nouvelle « expérience dans un cadre nouveau » [49]. En effet, c'est uniquement à partir d'un état de non intégration (ou formlessness) que peut jaillir, de manière créative, le «geste spontané ». La fonction de l'analyste consiste alors à en réfléchir, tel le visage d'une mère, l'absolue singularité. Cette orientation de la cure explique l'extraordinaire insistance de Winnicott sur la question du jeu.

Cette promotion winnicottienne du self induit un changement de perspective psychanalytique - non seulement par rapport à l'ego-psychology, même si ceci est rarement souligné, mais également par rapport à Freud et Klein.

Premièrement, elle suppose, en effet, une évidente distanciation vis-à-vis de la promotion, par l'ego-psychology, d'un moi fort, et notamment vis-à-vis de tout ce qu'une telle conception de la cure suppose d'adaptation à la réalité et de maîtrise du ça. Le self, au contraire, est ce cœur de 
la personnalité, rebelle à tout compromis et toute adaptation, à jamais introuvable (bien qu'on le cherche), vivant, isolé et silencieux. Le chercher implique de se dessaisir du moi, de sa fonction de synthèse et des défenses que celui-ci. À la limite, il est même possible de situer le moi fort du côté d'un éventuel faux self - ce que Winnicott semble parfois suggérer [50]. Selon cette perspective, on pourrait donc affirmer que l'orientation de la cure, pour Winnicott, subvertissant le mot d'ordre de l'ego-psychology, se laisserait résumer ainsi : Where id was, there (my/the) self shall be. Là où était du ça, du self devrait advenir.

Pourtant, une telle formulation se révèle inexacte. Non pas tant parce que le self ne serait pas au cœur de la cure, il semble bien que ce soit le cas, mais parce que la référence au ça (sans parler du surmoi) a, en réalité, été éclipsée dans l'approche winnicotienne comme nous l'avons déjà suggéré. Promouvoir le self, ce n'est pas seulement déconstruire l'ego de Hartmann 17 pour revenir à Freud, c'est en fait totalement déplacer le champ analytique lui-même selon une conception qu'on pourrait qualifier de «pré-analytique » ou, en tout cas, en opérant un pas de côté par rapport à la découverte freudienne - ce que Pontalis [52] et Green [53], parmi d'autres, ont déjà souligné.

Certes, chez Winnicott, le moi n'est pas tout à fait maître en sa demeure. Demeure une tension entre le faux et le vrai self, dans la mesure où le premier ignore qu'en son cœur vit le self dont il s'agit de partir en quête et auquel il conviendrait de laisser une part de jeu. Celui-ci n'a

17 Dans le cadre d'une psychologie du moi, Heinz Hartmann a en effet proposé de dissiper les ambiguïtés notionnelles et terminologiques attachées à l'Ich freudien en proposant d'isoler systématiquement l'ego, comme système psychique, des problématiques du self : «En psychanalyse, une distinction tranchée n'est pas toujours faite entre les termes de moi [ego], de soi [self] et de personnalité. Mais une différenciation de ces concepts paraît décisive si nous essayons de regarder systématiquement les problèmes posés à la lumière de la psychologie structurale de Freud. Quand on utilise le terme narcissisme, on semble souvent confondre deux couples d'opposés : le premier concerne le soi (self), la personne propre en opposition à l'objet, le second concerne le moi [comme système psychique] en opposition aux autres substructures de la personnalité. Cependant l'opposé d'investissement d'objet n'est pas investissement du moi [ego-calhexis] mais investissement de la personne propre, c'est-à-dire investissement de soi [self-calhexis]; lorsque nous parlons d'investissement de soi, cela n'implique pas que l'investissement est situé dans le ça, dans le moi, ou dans le surmoi [...]. On clarifierait alors les choses en définissant le narcissisme comme l'investissement libidinal non du moi mais du soi. » ([51], p. 84-85). 
pourtant plus rien de sexuel. La problématique des origines du vrai et du faux self résulte de la relation mère-enfant et non du conflit du moi avec la pulsion. D'ailleurs, il est significatif, à cet égard, que Winnicott s'inscrive ouvertement dans la lignée des travaux sur le self de Jung et Erikson, éclipsant l'un et l'autre la question du sexuel [47].

D'ailleurs, la notion de self ré-instaure également la problématique de la personne et de l'ipséité «the self... is the person who is me, who is only me, who has a totality based on the operation of the maturational process » [33]. Si la recherche du self ne laisse peut-être pas espérer des retrouvailles définitives avec ce dernier, elle vise néanmoins à ce que l'individu fasse « un » avec ce cœur vivant et silencieux, se sente réel dans une adhésion à soi et fasse l'expérience de sa «totalité » plutôt que de reconnaître le déchirement du moi freudien mû, à son insu, par des déterminations inconscientes. Bref, si le self n'a donc pas exactement toutes les caractéristiques du «moi » lacanien et s'est constitué pour penser la clinique « hors névrose », il offre néanmoins la promesse du « Je suis » plutôt qu'il ne nous expose au « Je est un autre »...

\section{Avec Lacan. Fonction imaginaire et consistance sociale du moi}

« Définir la nature du moi entraine très loin ». ([54], p. 11). C'est l'avertissement, et le programme, que Lacan fixait à l'auditoire de son séminaire de l'année 1954-1955 consacré exclusivement au moi dans la théorie de Freud et dans la technique de la psychanalyse. Le moi constituait ainsi un des angles d'attaque inauguraux de son retour à Freud et une condition préliminaire à la reprise des grands cas paradigmatiques freudiens.

\subsection{Politiques du moi}


En ce moment de son enseignement, Lacan s'inscrivait en effet décidément dans un contexte de bagarre impliquant la place de la psychanalyse dans la culture et dans son temps. L'enjeu était de dénoncer les impasses dialectiques d'une clinique analytique réglée sur le moi et ses valeurs supposées d'autonomie et d'adaptabilité telle que la psychanalyse nord-américaine d'alors pouvait en faire la promotion : «ce moi [i.e. autonome], ne vous y trompez pas : il ne s'agit que de celui du genre de psychanalyste qui vous attend $5^{\mathrm{e}}$ Avenue. Il vous adaptera à la réalité de son cabinet ». ([55], p. 353). Au contraire de la doctrine freudienne qui fait du moi une instance d'aliénation, toujours dévouée «à servir la loi d'un autre » ([56], p. 668), l'egopsychology promut la sphère d'un moi fort, autonome, émancipé des conflits, en somme conforme aux idéaux du self-made-man et tenant lieu de boussole dans la direction de la cure et dans les conditions de son achèvement. Pour Lacan, il n'y avait pas lieu de s'étonner que les psychanalystes instigateurs d'une telle idéologie soit des immigrés d'Europe centrale installés aux Etats-Unis du fait de la diaspora de la seconde guerre ([57], p. 206). Soucieux de préserver les prestiges de leur société d'origine dans une société d'accueil où les valeurs se sédimentent dans le capitalisme libéral, le moi constituait ainsi pour eux la préservation d'une unité de prestance idéale contre ce qu'ils pouvaient par ailleurs percevoir d'un morcellement social. Pourtant cette promotion du moi s'avérait finalement servir une conception individualiste et utilitariste de l'homme tout à fait syntone aux valeurs du nouveau monde. La nature d'asservissement du moi revenait pour ainsi dire par la fenêtre, là où on l'avait chassée par la porte.

En outre, Lacan, contre Daniel Lagache cette fois, entendait bien défaire la psychanalyse de ses adhérences douteuses à la psychologie. Car ce moi dit autonome, dont les travaux de 
Lagache se faisaient également le relai, est aussi bien ce «qui n'a plus servi que d'enseigne dans la psychologie elle-même dès qu'elle s'est voulue plus objective », concurremment à l'exploitation managériale de cette discipline, «spécialement dans ses usages de recrutement pour les emplois. » ([57], p. 205-206)

Qu'on n'aille cependant pas penser que ces querelles font désormais partie des archives de l'histoire de la psychanalyse en tant qu'elles seraient datées ou qu'elles ne concerneraient que l'espace étriqué de débats franco-français. L'actuelle domination d'un «individualisme de capacité » [58], notamment soutenu par les neurosciences cognitives, forgeant l'illusion d'un « homme augmenté » qui vise à transformer le handicap en «potentiel » et qui est indissociable d'une montée au zénith social d'une conception du «Soi », elle-même définie par tous les vocables de la connaissance spéculaire (de la représentations mentale aux techniques d'imagerie cérébrale), n'est sans doute qu'une forme peu originale de ce «nouvel Évangile » ([57], p. 206) que fut la promotion du moi autonome et fort au milieu du $\mathrm{XX}^{\mathrm{e}}$ siècle.

\subsection{L'espace géométrique du moi et son horizon}

Lacan opposait donc au moi ce qui lui est le plus étranger dans la structure, à savoir l'ordre du signifiant et de la parole, singulière, où un sujet a à se repérer quant au désir inconscient qui le fonde et l'oriente dans l'existence. On connait depuis ces principes opposés, élevés au classicisme dans une doxa lacanienne, qui délimitent d'une part le périmètre imaginaire du moi comme «série enveloppante» des identifications imaginaires ([57], p. 206), lieu de méconnaissance et de captation narcissique qui pousse chacun à croire savoir ce qu'il est, ce qu'il veut et ce qu'il pense, et d'autre part la transcendance du symbolique où s'articule, à l'insu 
du sujet, l'ordre des déterminations qui le dépassent mais auxquelles il consent sans le savoir et qui font donc de ce sujet un sujet divisé puisque, contrairement au moi, le sujet ne sait pas ce qu'il veut et ne se subjective pas.

Si Freud mettait déjà en exergue la spatialité du moi qu'il décrivait, comme on l'a vu, en terme de «surface », d' « enveloppe» ou encore de «strate» ou de «frontière », Lacan la développera avec son «Stade du miroir » puis en 1960, dans ses remarques à un rapport de Daniel Lagache justement, avec un modèle optique emprunté au physicien Henri Bouasse plus à même de rendre compte des effets de réfraction du clivage entre l'imaginaire et le symbolique dans la constitution des identifications et des idéaux du sujet. Dès Le stade du miroir, l'«imagerie du moi » ([59], p. 122) est conçue comme une unité synthétique, précipitée dans la Gestalt du corps propre et compensatoire d'une insuffisance imputable à la prématuration organique et adaptative du petit d'homme. L'«imago salutaire » ([59], p. 113) qui en résulte produit des « effets formatifs» en retour sur l'organisme. Cette forme leurrante primordiale, car « orthopédique » de la totalité de l'individu, puisqu'elle sert de point d'appui à une discordance que Lacan, recourant notamment à l'éthologie, conçoit d'abord comme neurologique puis comme inhérente à la structure même, entre dans la dialectique de l'identification à l'autre. Le moi, comme image de soi et image du corps, se construit à partir de l'image de l'autre, du semblable et des objets du monde. Et non pas l'inverse. Par conséquent, il s'agit de ne pas oublier que le moi et son assiette narcissique ont d'emblée une fonction socialisante qui trouve sa médiation dans le désir de l'autre. Il n'y a donc pas lieu d'opposer si facilement qu'on a tendance à le faire, narcissisme d'une part et lien social d'autre part. Par conséquent, on aurait tort de réduire le moi au palais des mirages qui esseule le sujet dans son narcissisme autant qu'il le leurre quant à son désir. 
Toutefois le sujet n'en est pas moins foncièrement décentré du moi. Il est sans qualité et constitue une sorte d'entité négative supposée à l'articulation signifiante dans l'inconscient. Il trouve d'ailleurs son complément, ou son semblant d'être, tout aussi négatif, dans un objet qui, lui, n'est pas supposé mais produit par la chaine signifiante, à savoir ce que Lacan appellera l'objet $a$; lequel vient incarner ce qui manque au sujet et cause ainsi son désir. Contrairement au moi qui s'élabore dans le stade du miroir et qui se supporte du set des identifications du sujet, l'objet $a$ est transpéculaire. Lui n'a pas d'image même si l'image est sa chasuble. D'où l'angoisse, quand l'objet et le désir qu'il porte affleurent, puisque le moi et sa fonction identifiante sont impuissants à fournir au sujet une image de lui-même d'où il pourrait se reconnaitre ici. Pourtant cette zone de non-moi est le plus intime du sujet «dont le corps imperceptible avait dévoré mon reflet » ([60], p. 52) s'exclame horrifié le protagoniste du Horla de Maupassant. Lacan le soutiendra un moment : c'est donc vers cet objet invisible, rebut de savoir dont l'analyste tient l'emploi, qu'une psychanalyse conduit, et non pas vers le moi qui le recouvre voire en empêche l'approche et le cerne. C'est d'ailleurs en quoi Lacan ne peut vraiment rejoindre ni Melanie Klein, ni D. W. Winnicott qui, malgré l'hommage qu'il peut leur rendre par ailleurs, sont restés inféodés à une approche binaire, et donc imaginaire, de la cure référée à la dyade mère-enfant. Même lorsque Melanie Klein envisage la formation du symbole dans la genèse du moi, c'est toujours un contenu imaginaire qui est promu selon Lacan ([61], p. 259). Quant à Winnicott, Lacan avait certes reconnu que sa conception du Self ne se réduisait pas au moi, et qu'il y avait là « une touche juste » en tant que le Self, au-delà ou en-deçà du moi, était un effet du signifiant 18. Cependant Lacan voyait en ce vrai self caché au plus profond de 
l'individu... la présence de Winnicott lui-même, de son Self à lui, et de sa conception de l'analyste comme devant répondre à tous les besoins du patient; condition nécessaire et suffisante d'un désaveu de l'acte analytique 19.

\subsection{Clinique du moi et émancipation de l'ego}

Il y aurait un intérêt à revenir sur la fonction que Lacan a réservée au moi dans une clinique différentielle des positions subjectives où le praticien ne peut faire l'économie de se demander où est le moi du sujet, pour mieux situer les paramètres du champ érotique de tel analysant dans le procès de la cure et dans l'approche de sa vérité. Que l'on cherche donc le moi du sujet hystérique en lieu et place d'un des acteurs de son intrigue raffinée. On le trouvera par exemple en la personne de Monsieur K pour Dora. Le moi y est le médium par l'intermédiaire duquel le sujet pose sa question sur le sexe, mettons : qu'est-ce qu'une femme ? Dans la stratégie obsessionnelle, le moi, dans le jeu, est l'ombre d'un autre, pair, camarade ou adversaire, qui est le support des exploits par où il fait ses preuves d'être vivant auprès d'un Autre, et répond ainsi à son incertitude sur l'existence. En outre, et au-delà du seul champ des névroses, le moi se décline «selon l'un de ces sentiments qui forment le spectre de l'irréalité, depuis les sentiments d'étrangeté, de déjà vu, de jamais vu, en passant par les fausses reconnaissances, les illusions de sosie, les sentiments de devinement, de participation, d'influence, les intuitions de signification, pour aboutir au crépuscule du monde...» ([62], p. 50-51). Mais ces phénomènes imaginaires justement, pour spectaculaires qu'ils soient, ne sont que des repères de la connaissance

19 Ibid, séance du 6 décembre 1967. 
spéculaire. Ils sont précieux pour la psychiatrie mais insuffisants pour la psychanalyse car ils ne disent rien de l'articulation du sujet au signifiant. Ils constituent certes une « sémiologie qui va de la plus subtile dépersonnalisation à l'hallucination du double » mais «ils n'ont eux-mêmes aucune valeur diagnostique quant à la structure du sujet (la psychotique entre autres) » ([63], p. 71). Néanmoins le moi conserve une place et une fonction cruciales car, même dans la psychose où l'énamoration adopte parfois une dimension prévalente, la relation du moi au semblable peut constituer le «signal central d'une médiation possible» ([64], p. 101) et participer d'une «relation aussi élevée que celle de l'amitié au sens où Aristote en fait l'essence du lien conjugal. » ([65], p. 574).

Pour conclure, une dernière remarque s'impose, nouant incidence clinique et parcours notionnel relatif au moi dans l'enseignement de Lacan. Dans le séminaire Le moi dans la théorie de Freud et dans la technique de la psychanalyse, Lacan semblait employer indifféremment les termes de «moi » et d'« ego ». Le terme d'« ego », à l'époque de ce séminaire, parait à la fois emprunté au mot latin « ego », et provenir ainsi de la lecture de Lacan de quelques philosophes, dont Descartes, et en même temps importé de la langue anglaise qui est justement celle des psychanalystes de l'ego-psychology avec lesquels Lacan débattait 20. Or, bien plus tardivement, soit dans le séminaire Le sinthome (1975-1976), Lacan reconvoque l'ego, cette fois en référence à la fonction de l'écriture chez l'écrivain irlandais James Joyce. Mais s'agit-il pour autant du même ego ? À partir de 1972, avec l'introduction du nœud borroméen, l'imaginaire, le symbolique et le réel entrent dans une relation d'équivalence. L'imaginaire n'est plus subordonné au symbolique, comme ce fut le cas dans les années 50. C'est la raison pour laquelle

20 Lévi-Strauss recoure au terme d' «Ego » dans certains passages de son ouvrage Les structures élémentaires de la parenté ([66]. Voir notamment pages 199, 208 et 225 ainsi que la figure 27. Lacan fait spécifiquement référence à cet usage dans « La science et la vérité » ([67], p. 862.) 
Lacan dira, en cette dernière période de son enseignement, qu' «il faut se briser à un nouvel imaginaire instaurant le sens »([68], p. 121). Ce nouvel imaginaire est celui-là même qui supporte une reconsidération du moi, de l'ego (qui ne parait plus tout à fait superposable au moi) et du narcissisme. Qu'est-ce que Lacan entend désormais par « ego » et qui parait dépourvu d'accent dépréciatif ? L'ego, dira-t-il d'abord comme pour souligner encore son voisinage avec le moi, est «ce que les anglais appellent l'ego, et les allemands l'Ich» ([68], p. 122). Cependant il ajoutera que, dans le cas de Joyce, l'ego «a rempli une fonction » et a joué « un tout autre rôle » ([68], p. 147) que celui qu'il joue habituellement ; sous-entendu peut-être, que celui qu'il joue quand il se confond avec le moi, à savoir cette «idée de soi comme corps [en tant qu'elle] a un poids. » ([68], p. 150). L'ego narcissique a donc rapport à quelque chose qui supporte le corps comme image. Jusque-là, rien de vraiment nouveau. Mais l'ego n'est pas réductible à ce rôle puisque chez Joyce, selon Lacan, l'ego a rempli une toute autre fonction. Il est comme tel à rapprocher d'une invention singulière, d'une solution symptomatique, qui passe chez Joyce par son usage particulier de la langue dans son écriture, et spécialement dans le fait que les mots, dans leur matérialité, disons comme corps réel, entrent dans un rapport d'homonymie avec ce qu'ils désignent, disons comme image ou comme signifié. C'est la raison de son style énigmatique. L'ego de Joyce, que Lacan nomme au passage d'ego «de fonction énigmatique » ([68], p. 153), est aussi qualifié par Lacan d'« ego correcteur» qui a une fonction réparatrice, comme tout symptôme, et qui permet spécialement à Joyce de restaurer un rapport manquant à l'imaginaire chez lui.

Dans cette perspective nouvelle, l'ego n'est plus vraiment le moi et il ne se limite pas à l'infatuation de la personne. Il intéresse toujours l'imaginaire, mais sans s'y cantonner puisqu'il implique un usage singulier du symbolique et du réel de la langue. Sa dimension d'invention 
propre évoque à la fois une conception inédite du narcissisme mais aussi du symptôme qui revisite, sans pour autant la contredire, la notion de moi telle que Lacan avait pu la forger quelques décennies auparavant.

\section{Conclusion}

Quel bilan tirer de ce parcours ? D'abord, que l'œuvre freudienne inaugure une série de problématiques inédites et formatrices pour le champ psychanalytique, liées à la situation du moi et de ses frontières, auquel doivent être tout d'abord assignées, dans l'esprit de Freud, ses triples limites 1) intrasubjectives, en tant que le moi est ouvert sur le ça mais séparé « de manière tranchée » du refoulé ; 2) extra-subjectives, en tant que le moi se constitue en s'opposant à la réalité du monde extérieur ; 3) intersubjectives, en tant que le moi se vit, dans son rapport à l'autre, comme séparé de l'objet tout en étant l'objet d'une série d'identifications constituantes.

Il se trouve que dans ces trois directions, les limites pensées par Freud sont profondément remaniées par les apports de Klein, de Winnicott et de Lacan, qui renouvèlent, chacun à leur manière, la problématique du moi et du non-moi selon qu'ils privilégient et prolongent telle ou telle des intuitions s'imposant au fondateur de la psychanalyse.

Schématiquement, Klein relance et aggrave le caractère problématique de la division faussement naturelle entre le moi et l'objet, en considérant que celle-ci ne peut se construire que dans une fantasmatique structurée par une série d'introjections et de projections, telles que les limites en sont finalement plus constituantes que constituées. Winnicott, lui, élargit la problématique en prenant en compte l'incidence décisive de l'environnement dans cette constitution, telle que c'est d'abord dans une aire d'illusion entre le sujet et l'objet que se déploie 
le sentiment de continuité donnant au moi son assise - ce dont témoigne du reste sa promotion du Self, et les figures cliniques inédites qui sont indexées à cette proposition inédite. Lacan enfin, recentre la problématique sur la structure d'aliénation imaginaire du moi vis-à-vis de son alter ego, contre laquelle peut assurément être mis en valeur l'ordre symbolique des déterminations inconscientes, mais avec laquelle il faut compter pour concevoir pleinement la fonction du moi quitte à promouvoir à l'occasion une réhabilitation de l'ego dans sa fonction réparatrice de sinthome.

Évaluer les grandes convergences entre ces quatre auteurs, et creuser par ailleurs leurs différences, permet certes de marquer l'originalité respective de chacune de leurs contributions à la théorie psychanalytique, mais aussi la continuité insistante pour la psychanalyse de certaines problématiques relatives à la séparation entre l'instance du moi et le sujet de l'inconscient, entre le moi et la réalité, entre le dehors et le dedans, entre le sujet et l'objet, entre l'ego et l'alter ego. En quoi c'est finalement l'éclatement du Ich freudien et la dispersion des problématiques qui s'ensuit chez les post-freudiens, privilégiant chacun telle ou telle piste en vertu des spécificités de leurs paradigmes cliniques, qui permettent de concevoir pleinement l'étendue du problème du moi dans la psychanalyse.

À l'heure d'une nouvelle floraison des discours de la «toute conscience», volontiers valorisés, sous l'égide des neurosciences cognitives, par la promotion des thématiques liées au « soi » et encouragés par l'idéal social d'autonomie et d'adaptation individuelle, sans doute que le rappel des problématiques psychanalytiques, destituant le moi de son hypercentralité, n'est pas sans résonner avec une troublante actualité. C'est que l'irritation intempestive de la « vexation » psychanalytique infligée au moi, s'agissant de sa vérité refoulée, conserve sa chronique modernité... 


\section{Déclaration de liens d'intérêts}

Les auteurs déclarent ne pas avoir de liens d'intérêts.

\section{Références}

[1] [1] Freud (S.). Une difficulté de la psychanalyse [1917]. In: Laplanche J, editor. CEuvres complètes, vol. XV. Paris: PUF; 2002. p. 41-52.

[2] [2] Freud S. Études sur l'hystérie [1895]. In: Laplanche J, editor. Euvres complètes, vol. II. Paris: PUF; 2009. p. 9-332.

[3] [3] Freud S. Nouvelle suite des leçons d'introduction à la psychanalyse [1933]. In: Laplanche J, editor. Euvres complètes, vol. XIX. Paris: PUF; 2004. p. 83-268.

[4] [4] Freud S. L'esquisse d'une psychologie scientifique [1895]. In: Laplanche J, editor. Lettres à Wilhelm Fliess 1887-1904. Paris: PUF; 2006.

[5] [5] Freud S. L'Interprétation du rêve [1899]. In: Laplanche J, editor. Euvres complètes, vol. IV. Paris: PUF; 2003. p. 7-704.

[6] [6] Lacan J. Variantes de la cure type [1955]. In: Écrits. Paris; Seuil. p. 323-62.

[7] [7] Freud S. Le trouble de vision psychogène [1911]. In: Laplanche J, editor. CEuvres complètes, vol. X. Paris: PUF; 2009. p. 177-86.

[8] [8] Freud S. Pour introduire le narcissisme [1914]. In: Laplanche J, editor. Euvres complètes, vol. XII. Paris: PUF; 2006. p. 213-45.

[9] [9] Freud S. Le moi et le ça [1923]. In: Laplanche J, editor. CEuvres complètes, vol. XVI. 
Paris: PUF; 2003. p. 255-301.

[10] [10] Freud S. Contribution à l'histoire du mouvement psychanalytique [1914]. In: Laplanche J, editor. Euvres complètes, vol. XII. Paris: PUF; 2006. p. 247-315.

[11] [11] Freud S. Malaise dans la civilisation [1929]. In: Laplanche J, editor. OEuvres complètes, vol. XII. Paris : PUF; 2006. p. 213-45.

[12] [12] Freud S. Formulations sur les deux principes de l'advenir psychique [1911]. In: Laplanche J, editor. Euvres complètes, vol. XI. Paris: PUF; 2005. p. 11-21.

[13] [13] Freud S. Pulsions et destins des pulsions [1915]. In: Laplanche J, editor. Euvres complètes, vol. XII. Paris: PUF; 2006. p. 213-45.

[14] [14] Freud S. La négation [1925]. In: Laplanche J, editor. Euvres complètes, vol. XVII. Paris: PUF; 1992. p. 165-72.

[15] [15] Freud S. L'inquiétant [1919]. In: Laplanche J, editor. Euvres complètes, vol. XV. Paris : PUF; 2002. p. 147-88.

[16] [16] Freud S. Fragment d'une analyse d'hystérie [1905]. In: Laplanche J, editor. CEuvres complètes, vol. VI. Paris: PUF; 2006. p. 183-302.

[17] [17] Freud S. Deuil et mélancolie [1915]. In: Laplanche J, editor. Euvres complètes, vol. XIII. Paris : PUF; 2005. p. 261-80.

[18] [18] Federn P. La psychologie du moi et les psychoses [1952]. In: Laplanche J, editor. Paris: PUF; 1979.

[19] [19] Freud S. De quelques mécanismes névrotiques dans la jalousie, la paranoïa et l'homosexualité. In: Laplanche J, editor. Euvres complètes, vol. XVI. Paris: PUF; 2010. p. $85-97$.

[20] [20] Freud S. Inhibition, symptôme et angoisse [1926]. In: Laplanche J, editor. CEuvres 
complètes, vol. XII. Paris: PUF; 1992. p. 183-302.

[21] [21] Klein M. Notes sur quelques mécanismes schizoïdes [1946]. In: Développements de la psychanalyse. Paris: PUF « Quadrige »; 2013. p. 274-300.

[22] [22] Klein M. Sur la théorie de l'angoisse et de la culpabilité [1948]. In: Klein M. et al, editors. Développements de la psychanalyse. Paris: PUF« Quadrige »; 2013. p. 254-73.

[23] [23] Klein M. Sur le développement du fonctionnement mental [1958]. In: Le transfert et autres écrits. Paris: PUF, coll. « Bibliothèque de psychanalyse »; 2007. p. 51-64.

[24] [24] Klein M. Quelques conclusions théoriques au sujet de la vie émotionnelle des bébés [1952]. In: Klein M. et al, editors. Développements de la psychanalyse. Paris: PUF « Quadrige »; 2013. p. 187-222.

[25] [25] Pontalis JB. Nos débuts dans la vie selon Melanie Klein. In : Après Freud. Paris: Gallimard; 1993. p. 181-202.

[26] [26] Baranger W. Position et objet dans l'œuvre de Melanie Klein. Paris: Erès; 1999. p. 107-27.

[27] [27] Klein M. Envie et Gratitude [1957]. Paris: Gallimard; 2008.

[28] [28] Klein M. L'importance de la formation du symbole dans le développement du moi [1930]. In: Essais de psychanalyse. Paris: Payot; 1968. p. 263-78.

[29] [29] Klein M. Contribution à la psychogenèse des états maniaco-dépressifs [1934]. In: Essais de psychanalyse. Paris: Payot; 1968. p. 311-40.

[30] [30] Klein M. Le deuil et ses rapports avec les états maniaco-dépressifs [1940]. In: Essais de psychanalyse. Paris: Payot; 1968. p. 341-69.

[31] [31] Klein M. Les influences mutuelles dans le développement du moi et du ça [1952]. In: Le transfert et autres écrits. Paris: PUF, coll. «Bibliothèque de psychanalyse »; 2007. p. 
$7-12$.

[32] [32] Winnicott DW. La préoccupation maternelle primaire [1956]. In: De la pédiatrie à la psychanalyse. Paris: Payot; 1969. p. 285-91.

[33] [33] Winnicott DW. Sur le corps et le Self [1970]. In: La crainte de l'effondrement et autres situations cliniques. Paris: Gallimard; 2000. p. 264-77.

[34] [34] Winnicott DW. L'angoisse liée à l'insécurité [1952]. In : De la pédiatrie à la psychanalyse. Paris: Payot; 1969. p. 198-202.

[35] [35] Klein M. Les racines infantiles du monde adulte. In: Envie et gratitude [1959]. Paris: Gallimard; 1959. p. 95-118.

[36] [36] Winnicott DW. La théorie de la relation parent-nourrisson [1960]. In: De la pédiatrie à la psychanalyse. Paris: Payot; 1969. p. 358-78.

[37] [37] Winnicott DW. Intégration du moi au cours du développement de l'enfant [1962]. In: Processus de maturation chez l'enfant : développement affectif et environnement. Paris : Payot ; 1970. p. 9-18.

[38] [38] Winnicott DW. Le développement affectif primaire [1945]. In: De la pédiatrie à la psychanalyse. Paris: Payot; 1969. p. 57-71.

[39] [39] Winnicott DW. Distorsion du moi en fonction du vrai et du faux « self» [1960]. In: Processus de maturation chez l'enfant : développement affectif et environnement. Paris: Payot; 1970. p. 115-32.

[40] [40] Winnicott DW. Les souvenirs de la naissance, le traumatisme de la naissance et l'angoisse [1949]. In : De la pédiatrie à la psychanalyse. Paris: Payot; 1969. p. 111-34.

[41] [41] Winnicott DW. La communication entre le nourrisson et la mère, et la mère et le nourrisson : comparaisons et contraste [1968]. In: Le bébé et sa mère. Paris: Payot; 1992. 
p. $125-46$.

[42] [42] Winnicott DW. Objets transitionnels et phénomènes transitionnels [1951]. In: Jeu et réalité. Paris: Gallimard; 1975. p. 27-64.

[43] [43] Winnicott DW. Un rêve de Winnicott [1963]. In: La crainte de l'effondrement et autres situations cliniques. Paris : Gallimard ; 2000. p. 243-45.

[44] [44] Winnicott DW. L'usage de l'objet et le mode de relation à l'objet au travers des identifications [1969]. In: La crainte de l'effondrement et autres situations cliniques. Paris: Gallimard; 2000. p. 231-42.

[45] [45] Winnicott DW. Les aspects métapsychologiques et cliniques de la régression au sein de la situation analytique [1954]. In: De la pédiatrie à la psychanalyse. Paris : Payot ; 1969. p. 250-67.

[46] [46] Winnicott DW. L'agressivité et ses rapports avec le développement affectif [19501955]. In : De la pédiatrie à la psychanalyse. Paris: Payot; 1969. p. 150-68.

[47] [47] Winnicott DW. De la communication et de la non-communication [1963]. In: Processus de maturation chez l'enfant : développement affectif et environnement. Paris: Payot; 1970. p. 151-68.

[48] [48] Winnicott DW. Psycho-Somatic disorder [1964]. In: Psychoanalytic explorations. Londres: Karnac;1989. p. 103-14.

[49] [49] Winnicott DW. Jouer. L'activité créative et la quête du soi [1971]. In: Jeu et réalité : l'espace potentiel. Paris: Gallimard; 1975. p. 108-26.

[50] [50] Winnicott DW. Les formes cliniques du transfert [1955-56]. In: De la pédiatrie à la psychanalyse. Paris: Payot; 1969. p. 279-84.

[51] [51] Hartmann H. Comments on the psychoanalytic theory of the ego. In: Psychoanalytic 
study of the child $1950 ; 5(1): 74-96$.

[52] [52] Pontalis JB. Naissance et reconnaissance du «soi »: pour introduire à l'espace potentiel. In: Entre le rêve et la douleur. Paris: Gallimard; 1977. p. 159-89.

[53] [53] Green A. Object(s) and subject. In: André Green at the squiggle foundation. Londres: Karnac; 2000. p. 17-37.

[54] [54] Lacan J. Le séminaire Livre II, Le moi dans la théorie de Freud et dans la technique de la psychanalyse. Paris: Seuil; 1978.

[55] [55] Lacan J. De la psychanalyse dans ses rapports à la réalité [1968]. In: Autres écrits. Paris: Seuil; 2001. p. 351-60.

[56] [56] Lacan J. Remarque sur le rapport de Daniel Lagache [1961]. In: Écrits. Paris: Seuil; 1966. p. $647-84$.

[57] [57] Lacan J. Réponses à des étudiants en philosophie [1966]. In: Autres écrits. Paris: Seuil; 2001. p. 203-12.

[58] [58] Ehrenberg A. La mécanique des passions : cerveau, comportement, société. Paris: Odile Jacob; 2018.

[59] [59] Lacan J. L’agressivité en psychanalyse [1948]. In: Écrits. Paris: Seuil; 1966. p. 10124.

[60] [60] Maupassant G. de. Le Horla et autres nouvelles fantastiques. Paris: Pocket; 2006.

[61] [61] Lacan J. Le séminaire Livre VI, Le désir et son interprétation. Paris: La Martinière; 2013.

[62] [62] Lacan J. Les complexes familiaux dans la formation de l'individu [1938]. In: Autres écrits. Paris: Seuil; 2001. p. 23-84.

[63] [63] Lacan J. De nos antécédents. In: Écrits. Paris: Seuil ; 1966. p. 65-72. 
[64] [64] Lacan J. Le séminaire Livre III, Les psychoses. Paris: Seuil; 1981.

[65] [65] Lacan J. D'une question préliminaire à tout traitement possible de la psychose [1959]. In: Écrits. Paris: Seuil ; 1966. p. 531-84.

[66] [66] Lévi-Strauss C. Les structures élémentaires de la parenté. Paris: Mouton \& Co; 1967.

[67] [67] Lacan J. La science et la vérité [1966]. In: Écrits. Paris: Seuil ; 1966. p. 855-78.

[68] [68] Lacan J. Le séminaire Livre XXIII, Le sinthome. Paris: Seuil; 2005. 